\title{
Dynamic Changes in Ultrastructure of the Primary Cilium in Migrating Neuroblasts in the Postnatal Brain
}

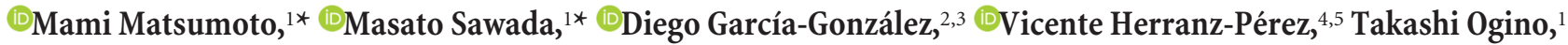 \\ 붕 Buy Bayen, ${ }^{6,7}$ Truc Quynh Thai, ${ }^{6,8}$ Keishi Narita, ${ }^{9}$ Natsuko Kumamoto, ${ }^{10}$ Shinya Ugawa, ${ }^{10}$ Yumiko Saito, ${ }^{11}$ \\ Sen Takeda, ${ }^{9}$ Naoko Kaneko, ${ }^{1}$ Konstantin Khodosevich, ${ }^{3}$ Hannah Monyer, ${ }^{2}$ José Manuel García-Verdugo, ${ }^{4}$ \\ Nobuhiko Ohno, ${ }^{6,12}$ and ${ }^{\circ}$ Kazunobu Sawamoto ${ }^{1,13}$ \\ ${ }^{1}$ Department of Developmental and Regenerative Neurobiology, Institute of Brain Science, Nagoya City University Graduate School of Medical Sciences, \\ Nagoya 467-8601, Japan, ${ }^{2}$ Department of Clinical Neurobiology, Medical Faculty of Heidelberg University and German Cancer Research Center, 69120 \\ Heidelberg, Germany, ${ }^{3}$ Biotech Research \& Innovation Centre, University of Copenhagen, 2200 Copenhagen, Denmark, ${ }^{4}$ Laboratory of Comparative \\ Neurobiology, Cavanilles Institute, University of Valencia, CIBERNED, 46980, Valencia, Spain, ${ }^{5}$ Predepartamental Unit of Medicine, Faculty of Health \\ Sciences, Universitat Jaume I, 12071 Castelló de la Plana, Spain, ${ }^{6}$ Division of Neurobiology and Bioinformatics, National Institute for Physiological Sciences, \\ Okazaki 444-8787, Japan, ${ }^{7}$ Department of Anatomy, Faculty of Medicine, University of Medicine and Pharmacy at Ho Chi Minh City, Ho Chi Minh City \\ 70000, Vietnam, ${ }^{8}$ Department of Histology-Embryology-Genetics, Faculty of Basic Medical Sciences, Pham Ngoc Thach University of Medicine, Ho Chi \\ Minh City 70000, Vietnam, ' Department of Anatomy and Cell Biology, Faculty of Medicine, University of Yamanashi, Chuo 409-3898, Japan, ${ }^{10}$ Department \\ of Anatomy and Neuroscience, Nagoya City University Graduate School of Medical Sciences, Nagoya 467-8601, Japan, ${ }^{11}$ Graduate School of Integrated \\ Sciences for Life, Hiroshima University, Hiroshima 739-8521, Japan, ${ }^{12}$ Department of Anatomy, Division of Histology and Cell Biology, Jichi Medical \\ University, School of Medicine, Shimotsuke 329-0498, Japan, and ${ }^{13}$ Division of Neural Development and Regeneration, National Institute for Physiological \\ Sciences, Okazaki 444-8585, Japan
}

New neurons, referred to as neuroblasts, are continuously generated in the ventricular-subventricular zone of the brain throughout an animal's life. These neuroblasts are characterized by their unique potential for proliferation, formation of chain-like cell aggregates, and long-distance and high-speed migration through the rostral migratory stream (RMS) toward the olfactory bulb (OB), where they decelerate and differentiate into mature interneurons. The dynamic changes of ultrastructural features in postnatal-born neuroblasts during migration are not yet fully understood. Here we report the presence of a primary cilium, and its ultrastructural morphology and spatiotemporal dynamics, in migrating neuroblasts in the postnatal RMS and OB. The primary cilium was observed in migrating neuroblasts in the postnatal RMS and OB in male and female mice and zebrafish, and a male rhesus monkey. Inhibition of intraflagellar transport molecules in migrating neuroblasts impaired their ciliogenesis and rostral migration toward the OB. Serial section transmission electron microscopy revealed that each migrating neuroblast possesses either a pair of centrioles or a basal body with an immature or mature primary cilium. Using immunohistochemistry, live imaging, and serial block-face scanning electron microscopy, we demonstrate that the localization and orientation of the primary cilium are altered depending on the mitotic state, saltatory migration, and deceleration of neuroblasts. Together, our results highlight a close mutual relationship between spatiotemporal regulation of the primary cilium and efficient chain migration of neuroblasts in the postnatal brain.

Key words: electron microscopy; live imaging; neuronal migration; olfactory bulb; primary cilium; rostral migratory stream

Significance Statement

Immature neurons (neuroblasts) generated in the postnatal brain have a mitotic potential and migrate in chain-like cell aggregates toward the olfactory bulb. Here we report that migrating neuroblasts possess a tiny cellular protrusion called a primary cilium. Immunohistochemical studies with zebrafish, mouse, and monkey brains suggest that the presence of the primary cilium in migrating neuroblasts is evolutionarily conserved. Ciliogenesis in migrating neuroblasts in the rostral migratory stream is suppressed during mitosis and promoted after cell cycle exit. Moreover, live imaging and 3D electron microscopy revealed that ciliary localization and orientation change during saltatory movement of neuroblasts. Our results reveal highly organized dynamics in maturation and positioning of the primary cilium during neuroblast migration that underlie saltatory movement of postnatalborn neuroblasts. 


\section{Introduction}

The ventricular-subventricular zone (V-SVZ), which lines the lateral walls of the lateral ventricles in the postnatal mammalian brain, harbors neural stem cells that continuously produce immature new neurons (neuroblasts) (Obernier and AlvarezBuylla, 2019). These neuroblasts are able to divide and migrate in chain-like aggregates toward the olfactory bulb $(\mathrm{OB})$ through the rostral migratory stream (RMS) (Luskin, 1993; Lois and AlvarezBuylla, 1994; Lois et al., 1996), and toward the lesioned cortex or striatum following brain injury (Arvidsson et al., 2002; Parent et al., 2002; Yamashita et al., 2006; Kreuzberg et al., 2010). Furthermore, the morphology of migrating neuroblasts is highly dynamic and controlled by multiple extrinsic and intrinsic cues (Khodosevich et al., 2009; Belvindrah et al., 2011; Nakamuta et al., 2017; Fujikake et al., 2018; Sawada et al., 2018). However, how ultrastructural features, such as the fine morphology of neurites and the position of internal organelles, contribute to neuroblast migration is not yet fully understood.

The primary cilium is a tiny cellular protrusion that contains signaling receptors on its membrane (Guemez-Gamboa et al., 2014; Malicki and Johnson, 2017), and regulates neural stem cell proliferation (Breunig et al., 2008; Han et al., 2008; AmadorArjona et al., 2011; Tong et al., 2014) and neuronal maturation (Kumamoto et al., 2012; Luo et al., 2015) in the postnatal brain. Primary cilia are reportedly present in cultured medial ganglionic eminence (MGE)-derived interneurons and cerebellar granule neurons (Baudoin et al., 2012; Trivedi et al., 2014). Both MGEderived interneurons and V-SVZ-derived neuroblasts show saltatory movement, in which the neuron extends a leading process, forms a swelling, and then translocates its soma toward the swelling (Bellion et al., 2005; Schaar and McConnell, 2005). The primary cilium extends from the basal body (Baudoin et al., 2012) and moves forward into the proximal leading process before somal translocation during saltatory movement (Trivedi et al., 2014). KO mice for the ciliary intraflagellar transport (IFT) molecules Kif3a or Ift88, or the ciliary small GTPase Arl13b, showed defective migration, morphogenesis, and connectivity of MGEderived interneurons (Baudoin et al., 2012; Higginbotham et al., 2012; Guo et al., 2017). Kif3a knockdown (KD) also disrupted migration and differentiation of embryonic pyramidal neurons (Chen et al., 2019). However, the primary cilium in postnatal-

Received June 16, 2019; revised 0ct. 8, 2019; accepted 0ct. 24, 2019.

Author contributions: M.M., M.S., D.G.-G., V.H.-P., K.N., N. Kumamoto, S.U., Y.S., S.T., N. Kaneko, K.K., H.M., J.M.G.-V., N.O., and K.S. designed research; M.M., M.S., D.G.-G., V.H.-P., T.O., H.B.N., T.Q.T., K.K., and N.O. performed research; M.M., D.G.-G., V.H.-P., T.O., N. Kaneko, H.M., J.M.G.-V., N.O., and K.S. analyzed data; M.M., M.S., D.G.-G., K.K., and K.S. wrote the paper; K.N., N. Kumamoto, S.U., Y.S., and S.T. contributed unpublished reagents/analytic tools.

This work was supported by Japan Agency for Medical Research and Development Research Grants JP19bm0704033 to K.S., JP19gm1210007 to K.S., and JP19jm0210060 to M.S., N. Kaneko, and K.S.; Japan Society for the Promotion of Science KAKENHI 26250019, 15H01384, 15H01217, 16H06280, 17H01392, 17H05512, 17H05750, 19H04757, 19 H04785, and 18 KK0213 to K.S., 26830014 and 18 K14823 to M.S., and 16J00373 to M.M.; Japan Society for the Promotion of Science Program for Advancing Strategic International Networks to Accelerate the Circulation of Talented Researchers S2704 to M.S., V.H-P., N. Kaneko, J.M.G-V., and K.S.; Bilateral Open Partnership Joint Research Projects to K.S.; Grant-in-Aid for Research at Nagoya City University to K.S.; Cooperative Study Programs of National Institute for Physiological Sciences to K.S.; the Mitsubishi Foundation to K.S.; the Canon Foundation to K.S.; the Takeda Science Foundation to K.S. and M.S.; Spanish Ministry of Science, Innovation and Universities PCI2018093062 to V.H-P. and J.M.G-V.; and Independent Research Fund Denmark 8020-00083B to K.K. M.M. was supported by a Japan Society for the Promotion of Science Fellowship. We thank Joseph G. Gleeson, Masahiro Yamaguchi, Hiroyuki Miyoshi, Joshua J. Breunig, Kozo Kaibuchi, Tatsunori Seki, and Shaoyu Ge for materials; Hisashi Oishi, Hiroshi Takase, Mikio Furuse, Shigeyuki Magi, and Kagayaki Kato for technical support; and Yoichi Kato, Yusuke Hirabayashi, and Sawamoto laboratory members for discussions.

The authors declare no competing financial interests.

*M.M. and M.S. contributed equally to this work.

Correspondence should be addressed to Kazunobu Sawamoto at sawamoto@med.nagoya-cu.ac.jp.

https://doi.org/10.1523/JNEUROSCI.1503-19.2019

Copyright $(2019$ the authors born neuroblasts migrating in chains in the RMS has not been investigated.

Primary cilium ultrastructure and dynamics in mature cells have been intensively studied. Transmission electron microscopy (TEM) analyses have revealed ultrastructural features of the primary cilium, including nine pairs of microtubules (MTs) (the axoneme) and a basal body with transition fibers and basal feet (Sorokin, 1962). Time-lapse imaging studies using fluorescent protein-fused Arl13b have demonstrated the dynamic behavior of the primary cilium (Higginbotham et al., 2012; Trivedi et al., 2014; Ford et al., 2018). In addition to these methodologies, new EM techniques that acquire high-resolution ultrastructural images in three dimensions have recently been used for primary cilium analysis (Paridaen et al., 2013; David et al., 2014; Buskin et al., 2018; Insinna et al., 2019). Serial block-face scanning electron microscopy (SBF-SEM) is one of the 3D-EM techniques that enable analysis of both cell morphology and subcellular organelles simultaneously at the ultrastructure level (Briggman and Bock, 2012; Denk et al., 2012; Ohno et al., 2015). SBF-SEM has been applied to the study of morphological changes in the primary cilium during mitosis in embryonic neuroepithelial cells (Paridaen et al., 2013). Therefore, SBF-SEM may also be useful for studying the morphology of the primary cilium in the V-SVZderived neuroblasts migrating in the RMS.

In this study, we examined the morphology and dynamics of the primary cilium in V-SVZ-derived neuroblasts migrating in the postnatal RMS and OB. Using time-lapse imaging, TEM, and SBF-SEM, we found that the localization and orientation of the primary cilium in migrating neuroblasts are altered during proliferation and saltatory movement. Our results suggest that the spatiotemporal regulation of the primary cilium is related to the efficient chain migration of postnatal-born neuroblasts.

\section{Materials and Methods}

Animals. WT male and female C57BL/6J mice were purchased from Japan SLC (RRID:IMSR_JAX:000664). Male and female GFP::Centrin2 (GFP::Cent2) mice (RRID:MGI:3793423) were described previously (Higginbotham et al., 2004), as were male 5HT3A-EGFP mice (Inta et al., 2008). Male R26-tdTomato mice (stock \#7914, The Jackson Laboratory; RRID:IMSR_JAX:007914) (Madisen et al., 2010) were provided by Dr. Masahiro Yamaguchi (Kochi Medical School). WT male and female AB (RRID:ZIRC_ZL1) zebrafish (Danio rerio) (3-8 months old) were obtained from the Zebrafish International Resource Center, and maintained with standard procedures as reported previously (Kishimoto et al., 2011,2013; Ogino et al., 2016). The 1-year-old male rhesus monkey brain was obtained from EUPRIM-Net biobank (www.euprim-net.eu). All experiments involving live animals were performed in accordance with the guidelines and regulations of Nagoya City University and Heidelberg University.

Viral vectors and plasmids. To generate $\mathrm{pENTR}$-GFP::Centrin2-IRESCre, a GFP::Centrin2 fragment was amplified from pEGFP-Cent2 (provided by Dr. Shaoyu Ge, Stony Brook University) by PCR and inserted into the SalI site of pENTR-IRES-Cre (Jinnou et al., 2018; Sawada et al., 2018). To generate pENTR-D-TOPO-dnKif3A, a dnKif3A fragment was amplified from pTetOn-dTomato-F2A-dnKif3A (Kumamoto et al., 2012) by PCR and inserted into the pENTR-D-TOPO vector. Gateway cloning technology (Invitrogen) was used to generate the following expression vectors: CSII-EF-GFP::Centrin2-IRES-Cre, CSII-EF-dnKif3AIRES-Venus, and pCAGGS-dnKif3A. To generate CSII-CMV-Arl13b:: Venus, an Arl13b::Venus fragment was inserted into the Eco47III site of CSII-CMV-MCS-IRES2-Bsd. CSII lentiviral vectors were provided by Dr. Hiroyuki Miyoshi (RIKEN Tsukuba BioResource Center). To generate lentiviral particles, lentiviral vectors and packaging vectors (pCAGHIVgp and pCMV-VSV-G-RSV-Rev) were transfected into HEK293T cells. Three days after transfection, the culture supernatants were con- 
centrated by centrifuging at $8000 \mathrm{rpm}$ at $4^{\circ} \mathrm{C}$ for $16 \mathrm{~h}$ using an MX-307 refrigerated microcentrifuge (Tomy).

To obtain pCDH-CMV-5HT6::GFP and pCDH-CMV-ACIII::GFP lentiviral vectors, 5HT6::GFP (Addgene, \#35624) and ACIII::GFP (kindly provided by Dr. Joshua J. Breunig) (Guadiana et al., 2013) cassettes were subcloned into the lentiviral vector $\mathrm{PCDH}-\mathrm{CMV}$ (the original pCDH plasmid was from System Bioscience). Recombinant viruses were produced and titrated as done previously (Khodosevich et al., 2012, 2013). Briefly, HEK293 cells were transfected with the viral backbone vector together with lentiviral helper plasmids (pMDLgpRRE, pRSVRev, and pMD2.G), and viral particles were collected by ultracentrifugation $(\sim 120,000 \times g$; Beckman $) 48-60 \mathrm{~h}$ after transfection. The viral pellet was resuspended in D-PBS, aliquoted, and stored at $-80^{\circ} \mathrm{C}$ until use. Viral titers were determined by infecting HEK293 cells in 96-well plates across 5 orders of dilutions. Before injection, viral titer was adjusted to $2 \times 10^{7}$ to $2 \times 10^{8}$ fluorescent plaque-forming units $(\mathrm{fpfu}) / \mathrm{ml}$.

For an IFT88-KD experiment using retrovirus, pU6-shRNA IFT88EFla-EGFP (provided by Dr. Shaoyu Ge, Stony Brook University) was used as reported previously (Kumamoto et al., 2012). To generate retroviral particles, pU6-shRNA IFT88-EF1a-EGFP and packaging vectors (pCAG-HIVgp and pCMV-VSV-G-RSV-Rev) were transfected into HEK293gp cells. Three days after transfection, the culture supernatants were concentrated by centrifugation as described above. To visualize 5HT3A localization in migrating neuroblasts, a retrovirus expressing 5HT3A::GFP (MMLV-RSV-EGFP-5HT3A) was used as reported previously (García-González et al., 2017). Recombinant retroviruses were produced by transfection of HEK293 cells with retroviral vectors and helper plasmids (pGP and pMD2.G), and viral particles were collected by ultracentrifugation as described above. The viral pellet was resuspended in D-PBS, and viral titer was adjusted to $2 \times 10^{7}$ to $2 \times 10^{8} \mathrm{fpfu} / \mathrm{ml}$ before injection.

To generate pEGFP-N1-SSTR3::GFP, an SSTR3::GFP fragment was inserted into the HindIII/KpnI sites of pEGFP-N1 (Clontech). To generate pdTomato-C1-Cent2 (dTomato::Cent2), EGFP cDNA in pEGFPCent2 (Kumamoto et al., 2012) was replaced with dTomato cDNA. pcDNA3.1-EB3-mEGFP (EB3::GFP) was provided by Dr. Kozo Kaibuchi (Nagoya University) (Watanabe et al., 2015).

In vivo viral infection and transplantation. Injection of lentiviral suspension into the V-SVZ of adult WT and R26-tdTomato mice was performed as described previously (Sawamoto et al., 2006) with modification. A $2 \mu \mathrm{l}$ volume of lentiviral suspension was stereotaxically injected into the anterior V-SVZ (1.0 mm anterior, 1.0, 1.1, $1.2 \mathrm{~mm}$ lateral to bregma and 1.8-2.3 mm deep) of male adult WT and R26-tdTomato mice. For injection of retrovirus encoding Ift 88 shRNA, $2 \mu$ l of retroviral suspension was stereotaxically injected into the anterior V-SVZ $(1.0 \mathrm{~mm}$ anterior, 1.0, 1.1, and $1.2 \mathrm{~mm}$ lateral to bregma and 1.8-2.3 mm deep) of male adult WT mice. For injection of retrovirus expressing 5HT3A::GFP, $1 \mu \mathrm{l}$ of retroviral suspension was stereotaxically injected into the anterior V-SVZ (0.6 mm anterior, $1.2 \mathrm{~mm}$ lateral to bregma, and $1.7 \mathrm{~mm}$ deep) of P20 male WT mice, as reported previously (García-González et al., 2017).

Transplantation of neonatal V-SVZ cells into the V-SVZ of adult WT mice was performed as described previously (Sawada et al., 2018) with modification. The V-SVZ tissues from postnatal day 0-1 (P0-P1) WT mice were dissected and dissociated with trypsin-EDTA (Invitrogen). The cells were washed with L-15 medium (Invitrogen) containing 40 $\mu \mathrm{g} / \mathrm{ml}$ DNase I (Roche Diagnostics) and transfected with $2.0 \mu \mathrm{g}$ pEGFPN1-SSTR3::GFP using the Amaxa Nucleofector II system (Lonza Walkersville). The transfected cells were resuspended in L-15 medium and stereotaxically injected into the anterior V-SVZ (1.0 $\mathrm{mm}$ anterior, 1.0, 1.1, $1.2 \mathrm{~mm}$ lateral to bregma, and $1.8-2.3 \mathrm{~mm}$ deep) of adult male WT mice.

In vitro time-lapse imaging of migrating neuroblasts. In vitro culture of V-SVZ-derived neuroblasts was performed as described previously (Ota et al., 2014; Sawada et al., 2018). The V-SVZ tissues were dissected from P0-P1 male and female WT mice and dissociated with trypsin-EDTA (Invitrogen). The cells were washed in L-15 medium (Invitrogen) containing $40 \mu \mathrm{g} / \mathrm{ml}$ DNase I (Roche Diagnostics) and transfected with plasmids using the Amaxa Nucleofector II system (Lonza Walkersville). The transfected cells were recovered in RPMI-1640 medium (Thermo Fisher
Scientific), allowed to aggregate, and then embedded in 60\% Matrigel (BD Biosciences) in L-15 medium. The cell aggregates were cultured in Neurobasal medium containing 2\% NeuroBrew-21 (Invitrogen), 2 mm L-glutamine (Invitrogen), and $50 \mathrm{U} / \mathrm{ml}$ penicillin-streptomycin (Invitrogen) for $2 \mathrm{~d}$.

Four-dimensional time-lapse images of the basal body and primary cilium in migrating neuroblasts were captured at $2 \mathrm{~min}$ and $0.6 \mu \mathrm{m}$ intervals using an LSM880 laser-scanning confocal microscope (Carl Zeiss) and a $63 \times$ oil-immersion objective lens. Time-lapse images of DsRed-expressing migrating neuroblasts in chains were captured at 1 min intervals using an inverted light microscope (Axio-Observer, Carl Zeiss) equipped with a Colibri light-emitting diode light system and a $40 \times$ objective lens, as reported previously (Sawada et al., 2018). Behaviors of migrating neuroblasts and GFP::Cent $2{ }^{+}$basal body were tracked using the ImageJ manual tracking tool. The resting phase was defined as a migration speed $<12 \mu \mathrm{m} / \mathrm{h}$ (Jinnou et al., 2018). Three-dimensional reconstructions of migrating neuroblasts were performed using Imaris (Carl Zeiss) as reported previously (Hikita et al., 2014), based on the faint cytosolic $\mathrm{GFP}^{+}$(see Fig. $2 B$ ) or dTomato ${ }^{+}$(see Fig. $2 G$ ) signals.

For EB3::GFP imaging, time-lapse images of EB3::GFP ${ }^{+}$dots in migrating neuroblasts were captured at $4 \mathrm{~s}$ intervals using an LSM880 laserscanning confocal microscope (Carl Zeiss) and a $63 \times$ oil-immersion objective lens, as reported previously (Sawada et al., 2018) with modification. All the EB3:: $\mathrm{GFP}^{+}$dots pursuable for $8 \mathrm{~s}$ (three sequential frames) were tracked using the ImageJ manual tracking tool, and their moving speed was measured using the ImageJ manual tracking tool.

Immunohistochemistry and image acquisition. Immunohistochemistry in mouse brain sections was performed as described previously (Ota et al., 2014; Sawada et al., 2018). Briefly, the adult brains were fixed by transcardiac perfusion with $4 \%$ PFA in $0.1 \mathrm{M} \mathrm{PB}$, and postfixed overnight at $4^{\circ} \mathrm{C}$ in the same fixative. Fifty-micrometer-thick floating coronal and sagittal sections were made using a vibratome (VT-1200S, Leica Microsystems) and incubated for $30 \mathrm{~min}$ at room temperature (RT) in $10 \%$ normal donkey serum and $0.2 \%$ Triton X-100 in PBS (blocking solution). These sections were incubated with primary antibodies in blocking solution overnight at $4^{\circ} \mathrm{C}$, and with AlexaFluor-488/-568/-647conjugated secondary antibodies (1:1000, Invitrogen) for $2 \mathrm{~h}$ at RT in blocking solution. The following primary antibodies were used: rabbit anti-adenylate cyclase III (ACIII) (1:200, sc-588, Santa Cruz Biotechnology; RRID:AB_630839); mouse IgG anti-Arl13b (1:1000, 75-287, NeuroMab; RRID:AB_2341543); rabbit anti-doublecortin (Dcx) (1:1000, 4604S, Cell Signaling Technology; RRID:AB_10693771); rabbit antiDsRed (1:1000, 632496, Clontech; RRID:AB_10013483); rabbit antiGFP antibody (1:1000, A6455, Invitrogen; RRID:AB_221570); rat anti-GFP (1:1000, 04404-84, Nacalai Tesque; RRID:AB_10013361); rabbit anti-calbindin (CB) (1:1000, CB38, Swant; RRID:AB_2721225); rabbit anti-calretinin (1:1000, 7697, Swant; RRID:AB_2619170); rabbit anti-Ki67 (1:1000, Leica Microsystems, NCL-Ki67p; RRID:AB_442102); rabbit anti-parvalbumin (PV) (1:1000, PV235, Swant; RRID:AB_10000343); mouse IgG anti-phospho-histone H3 (pHH3) (1:1000, 9706, Cell Signaling Technology; RRID:AB_331748); and sheep anti-tyrosine hydroxylase (TH) (1: 1000, AB1542, Millipore; RRID:AB_90755) antibodies. Nuclei were stained with Hoechst 33342 (1:5000, Invitrogen)

Immunohistochemistry in zebrafish brain sections was performed as described previously (Kishimoto et al., 2011; Ogino et al., 2016). The fish were anesthetized with tricaine. The brain was dissected and fixed with $4 \%$ PFA in $0.1 \mathrm{M} \mathrm{PB}$ overnight at $4^{\circ} \mathrm{C}$. Fifty-micrometer-thick floating coronal sections were made using a vibratome (VT-1200S, Leica Microsystems) and incubated for $30 \mathrm{~min}$ at RT in 10\% normal donkey serum and $0.2 \%$ Triton X-100 in PBS (blocking solution). These sections were incubated with primary antibodies in blocking solution overnight at $4^{\circ} \mathrm{C}$, and with AlexaFluor-488/-568-conjugated secondary antibodies (1:500, Invitrogen) for $2 \mathrm{~h}$ at $\mathrm{RT}$ in blocking solution. The following primary antibodies were used: mouse IgM anti-polysialylated form of neural cell adhesion molecule (PSA-NCAM) (1:1000, a kind gift from Dr. Tatsunori Seki, Tokyo Medical University) (Seki and Arai, 1991) and mouse IgG anti-acetylated tubulin (1:11000, Sigma-Aldrich; RRID:AB_477585). Nuclei were stained with Hoechst 33342 (1:5000, Invitrogen). 
Table 1. Numbers of neuroblasts examined in the SBF-SEM analyses ${ }^{a}$

\begin{tabular}{|c|c|c|c|c|c|c|c|}
\hline & \multirow[b]{2}{*}{ Data } & \multicolumn{5}{|c|}{ Ciliary types } & \multirow[b]{2}{*}{ Total } \\
\hline & & Centrioles & $\begin{array}{l}\text { Ciliary } \\
\text { vesicle }\end{array}$ & Procilium & $\begin{array}{l}\text { Nonextended } \\
\text { primary cilium }\end{array}$ & $\begin{array}{l}\text { Extended } \\
\text { primary cilium }\end{array}$ & \\
\hline \multirow[t]{3}{*}{ Results } & Neuroblast orientation & 一 & - & - & - & - & $418(88)$ \\
\hline & Leading-process extension phase & $22(0)$ & $23(0)$ & $10(0)$ & $82(0)$ & $6(0)$ & $143(0)$ \\
\hline & Swelling-formation phase & $10(0)$ & $14(0)$ & $28(0)$ & $33(0)$ & $22(0)$ & $107(0)$ \\
\hline \multirow[t]{3}{*}{ Fig. $5 F-H$ (Fig. $7 B, M)$} & Somal translocation phase & $9(0)$ & $11(0)$ & $8(0)$ & $44(0)$ & $8(0)$ & $80(0)$ \\
\hline & Leading-process extension phase & - & - & - & - & - & $15(0)$ \\
\hline & Swelling-formation phase & - & - & - & - & - & $15(0)$ \\
\hline Fig. $5 /$ & Somal translocation phase & - & - & - & - & - & $15(0)$ \\
\hline Results & Mitochondrial length & - & - & - & - & - & $18(0)$ \\
\hline \multirow[t]{2}{*}{ Fig. 50} & Mitosis & - & - & - & - & - & $1(0)$ \\
\hline & RMS & $41(0)$ & $48(0)$ & $46(0)$ & $159(0)$ & $36(0)$ & $330(0)$ \\
\hline \multirow[t]{2}{*}{ Fig. $7 A$} & $\mathrm{OB}$ & $1(1)$ & $2(2)$ & $1(1)$ & $7(7)$ & $14(14)$ & $25(25)$ \\
\hline & RMS & - & - & - & $159(0)$ & $36(0)$ & $195(0)$ \\
\hline Fig. $7 C$ & $\mathrm{OB}$ & - & - & - & $7(7)$ & $14(14)$ & $21(21)$ \\
\hline Fig. $7 D, E$ & Neuroblast & - & - & - & - & $28(0)$ & $28(0)$ \\
\hline Fig. $7 F, G$ & Astrocyte & - & - & - & - & $8(0)$ & $8(0)$ \\
\hline Fig. $7 H, I$ & Granule cell & - & - & - & - & $3(3)$ & $3(3)$ \\
\hline Fig. 7J,K & Presynaptic structure & - & - & - & - & $11(11)$ & $11(11)$ \\
\hline
\end{tabular}

${ }^{a}$ Values in parentheses indicate the numbers of neuroblasts with partial cell morphology (partial cells).

Immunohistochemistry in rhesus monkey brain sections was performed as described previously (García-González et al., 2017). Briefly, the brain was dissected, fixed with $4 \%$ PFA in $0.1 \mathrm{M} \mathrm{PB} 24 \mathrm{~h}$ at $4^{\circ} \mathrm{C}$, and cut into $0.5 \mathrm{~cm}$ slabs. These slabs were postfixed with $4 \%$ PFA in $0.1 \mathrm{M} \mathrm{PB}$, cryoprotected with $30 \%$ sucrose for $72 \mathrm{~h}$. Fifty-micrometer-thick coronal sections were prepared by cryostat (Leica Microsystems). These sections were incubated for $30 \mathrm{~min}$ at RT with 5\% BSA and 0.5\% Triton $\mathrm{X}-100$ in blocking solution, primary antibodies in blocking solution for $48 \mathrm{~h}$ at $4^{\circ} \mathrm{C}$, and AlexaFluor-488/-568-conjugated secondary antibodies in blocking solution for $24 \mathrm{~h}$ at $4^{\circ} \mathrm{C}$. The following primary antibodies were used: goat anti-Dcx antibody (1:250, sc-8066, Santa Cruz Biotechnology; RRID:AB_2088494) and mouse IgG anti-Arl13b (1:1000, 75287, NeuroMab; RRID:AB_2341543). Nuclei were stained with DAPI (1:5000, Thermo Fisher Scientific; RRID:AB_2307445).

Images of stained RMS and $\mathrm{OB}$ sections were acquired by scanning at $0.4 \mu \mathrm{m}$ (see Figs. $1,3 C, F$ ) or $2 \mu \mathrm{m}$ (see Fig. $3 A, B, D, E, G-I$ ) intervals using an LSM 700 confocal laser-scanning microscope (Carl Zeiss) with a $40 \times$ water-immersion objective lens. To investigate the positional relationship of the basal body and primary cilium in detail (see Fig. 1B), images of GFP::Cent $2^{+}$signals and Arl13b ${ }^{+}$signals were acquired by scanning at $0.4 \mu \mathrm{m}$ intervals using an LSM 880 Airyscan confocal laserscanning microscope with a $63 \times$ oil-immersion objective lens. For quantification of labeled cells in viral experiments (see Fig. 3), all the labeled cells observed in the RMS and OB in every section (dnKif3A) or every third section (IFT88-KD) were counted.

TEM. Sample preparation for TEM was performed as described previously (Kaneko et al., 2018). Adult male mouse brains were fixed by transcardiac perfusion with $2 \%$ PFA and $2.5 \%$ glutaraldehyde in $0.1 \mathrm{M}$ $\mathrm{PB}, \mathrm{pH} 7.4$, at $4^{\circ} \mathrm{C}$, and postfixed overnight at $4^{\circ} \mathrm{C}$ in the same fixative. These brains were cut into $200-\mu \mathrm{m}$-thick coronal sections using a vibratome (VT-1200S, Leica Microsystems) (anterior 2.0-2.3 mm from bregma). The sections were treated with $2 \%$ osmium tetroxide $\left(\mathrm{OsO}_{4}\right.$, Electron Microscopy Sciences) in $0.1 \mathrm{M} \mathrm{PB}, \mathrm{pH} 7.4$, at $4^{\circ} \mathrm{C}$, dehydrated with a graded series of ethanol, placed in propyleneoxide (Nisshin EM), and embedded in Durcupan resin for $72 \mathrm{~h}$ at $60^{\circ} \mathrm{C}$. Semithin sections (1.5- $\mu \mathrm{m}$-thick) were sequentially cut using an ultramicrotome (UC6, Leica Microsystems) with a diamond knife (histo, DiATOME), and stained with $1 \%$ toluidine blue, and sections of interest were chosen under a light microscope CX23 (Olympus). Ultrathin sections (60- to 70 -nm-thick) were sequentially cut from the embedded semithin sections using an ultramicrotome (UC6, Leica Microsystems) with a diamond knife (SYM2045, SYNTEK), and stained with $2 \%$ uranyl acetate in distilled water for $15 \mathrm{~min}$ and with modified Sato's lead solution for 5 min. TEM images of the primary cilium in neuroblasts were acquired using a JEM-1400plus (JEOL) fitted with a digital camera. Thirty-six neuroblasts were examined in this study.

SBF-SEM. Sample preparation, observation with SBF-SEM, and analyses of acquired data were performed as described previously with slight modifications (Nguyen et al., 2016; Thai et al., 2016; Kaneko et al., 2018; Sawada et al., 2018). Adult WT brains were fixed by transcardiac perfusion with $2.5 \%$ glutaraldehyde and $2 \% \mathrm{PFA}$ in $0.1 \mathrm{M} \mathrm{PB}, \mathrm{pH} 7.4$, at $4^{\circ} \mathrm{C}$, and postfixed overnight at $4^{\circ} \mathrm{C}$ in the same fixative. Fixed RMS tissues (anterior $2.0-2.3 \mathrm{~mm}$ from bregma) were treated with $2 \% \mathrm{OsO}_{4}$ and $1.5 \%$ potassium hexacyanoferrate in $\mathrm{PBS}$ for $1 \mathrm{~h}$ at $4^{\circ} \mathrm{C}, 1 \%$ thiocarbohydrazine for $20 \mathrm{~min}$ at $\mathrm{RT}, 2 \%$ aqueous $\mathrm{OsO}_{4}$ for $30 \mathrm{~min}$ at RT, and lead aspartate solution for $30 \mathrm{~min}$ at $65^{\circ} \mathrm{C}$. The RMS tissues were then dehydrated in a graded ethanol series, treated with dehydrated acetone, and embedded in Quetol 812 epoxy resin containing Ketjen black powder for $3 \mathrm{~h}$ at $95^{\circ} \mathrm{C}$ to ensure polymerization. SBF-SEM observation of the RMS and $\mathrm{OB}$ was performed using a Merlin and Sigma scanning electron microscope (Carl Zeiss) equipped with a $3 \mathrm{View}$ in-chamber ultramicrotome system (Gatan). Serial image sequences were $40.96 \times 40.96 \mu \mathrm{m}$ wide $(5.0 \mathrm{~nm} /$ pixel $)$ and $>80 \mu \mathrm{m}$ deep with $80 \mathrm{~nm}$ steps. Sequential images were processed using Fiji. In the image stacks, $\sim 2 \%$ of the total images were replaced by neighboring images because some parts of the images were contaminated by debris or corrupted during image acquisition. Our SBF-SEM analyses contained neuroblasts with whole and partial morphology ("whole cells" and "partial cells," respectively). "Partial cells," containing part of the nucleus, Golgi apparatus, and centrosome or basal body with ciliary structures, and having a leading process $>6$ $\mu \mathrm{m}$, were used for analyses of neuroblast orientation in the RMS (described in Results), ciliary classification (see Fig. 7A), and ciliary length (see Fig. 7C). Numbers of analyzed whole and partial cells in the SBFSEM analyses (see Figs. 5, 6, 7) are summarized in Table 1. Segmentation of the cell membrane, nuclei, centrioles, mitochondria, Golgi apparatus, and primary cilium were performed using Microscopy Image Browser (Belevich et al., 2016). Manual segmentation was done in every 2 nd-10th and interpolation tool was used for the other slices. Three-dimensional reconstruction of migratory neuroblasts and their subcellular organs was performed using Amira software (Maxnet) as reported previously (Kaneko et al., 2018; Sawada et al., 2018). Three-dimensional object files (.obj) obtained from Amira software were further processed by Blender software (https://www.blender.org/) and visualized as interactive 3D models by Sketchfab (https://sketchfab.com).

Experimental design and statistical analysis. Statistical analyses were performed using EZR (Kanda, 2013), as reported previously (Fujikake et al., 2018). The experiments were not randomized. Sample sizes were not predetermined but were chosen based on previous studies. All the nu- 
merical data, except for Figure $7 M$, were two-sided, and are shown as the mean \pm SEM. The normality of the data was analyzed by KolmogorovSmirnov test. For normally distributed data, equality of variances was examined by $F$ test, and a comparison of means between two independent groups was performed by unpaired $t$ test or Welch's $t$ test. For comparisons of means among three or more independent groups, oneway ANOVA followed by Tukey-Kramer test was applied. To analyze the dataset from multiple observations of individual samples, two-way repeated-measures ANOVA followed by post hoc Tukey-Kramer test or unpaired $t$ test between groups with Bonferroni correction was performed. For not normally distributed data, a comparison of medians between groups was performed by Mann-Whitney $U$ test, Kruskal-Wallis test followed by Steel-Dwass test, or Friedman's test followed by Wilcoxon signed rank test with Bonferroni correction. In Figure $7 M$, all the data indicate the probability, and they were compared by Fisher's exact test. $p<0.05$ was considered to be statistically significant.

\section{Results}

Expression of ciliary markers in migrating neuroblasts in the postnatal vertebrate RMS

To examine whether migrating neuroblasts in the postnatal RMS and $\mathrm{OB}$ present a primary cilium, we first stained brain sections for primary cilium marker Arl13b (Caspary et al., 2007) (Fig. $\left.1 A 1-B^{\prime}\right)$. To observe the basal body and a ciliary marker simultaneously, we studied $\mathrm{Dcx}^{+}$neuroblasts in transgenic mice expressing GFP-tagged Centrin-2 protein (GFP::Cent2) (Fig. $\left.1 A 1-A 2^{\prime}\right)$ or V-SVZ-derived neuroblasts in WT mice infected with the GFP::Cent2-expressing lentivirus (Fig. 1 B, $B^{\prime}$ ) (Higginbotham et al., 2004; Kumamoto et al., 2012). An Arl13b-positive $\left(\right.$ Arl13b $\left.{ }^{+}\right)$structure was observed in close proximity to one of the centrioles in the Dcx ${ }^{+}$neuroblasts in the RMS and OB, suggesting that it is associated with the basal body. Another marker for the primary cilium, ACIII (Bishop et al., 2007), is reported to be expressed in mature granule cells in the OB (Luo et al., 2015). ACIII was also detectable in migrating neuroblasts in the RMS (Fig. 1C) and mature interneurons in the external plexiform layer and glomerular layer of the OB (Fig. 1D-G). Moreover, fluorescent protein-tagged ciliary markers, such as Arl13b::Venus (Fig. 1H), ACIII::GFP (Fig. 1I), and 5HT6::GFP (Fig. 1J) (Brailov et al., 2000), accumulated in the perinuclear region of $\mathrm{Dcx}^{+}$neuroblasts in the RMS. These results suggest that the migrating neuroblasts possess a primary cilium in the postnatal RMS and OB.

A functional primary cilium expresses signaling receptors on its membrane (Malicki and Johnson, 2017; Wheway et al., 2018). To investigate the subcellular localization of ciliary receptors in migrating neuroblasts in the RMS, we introduced GFP-tagged serotonin receptor $3 \mathrm{~A}$ (5HT3A::GFP) or somatostatin receptor-3 (SSTR3::GFP) into migrating neuroblasts. These ciliary receptors colocalized with a ciliary marker (ACIII or Arl13b) (Fig. $1 K-M^{\prime}$ ), suggesting that $\mathrm{V}$-SVZ-derived migrating neuroblasts possess a functional primary cilium in the postnatal RMS.

Furthermore, we examined whether the expression of ciliary markers in migrating neuroblasts in the RMS is conserved in other vertebrates. We observed an acetylated tubulin-positive primary cilium in neuroblasts in the adult zebrafish RMS (Fig. $1 N, N^{\prime}$ ), and an Arl13b-positive primary cilium in the 1-year-old rhesus monkey RMS and OB (Fig. 1O- $P^{\prime \prime}$ ). Collectively, these results suggest that the $\mathrm{V}$-SVZ-derived migrating neuroblasts possess a primary cilium in the postnatal vertebrate brain.

\section{Dynamic changes in primary cilium localization during neuronal saltatory migration}

Previous EM studies have shown that the primary cilium is located at the cell surface at a distance from the nucleus, or in the perinuclear cytosol, in cultured MGE-derived interneurons (Baudoin et al., 2012). Furthermore, time-lapse imaging of Arl13b::Venus in cultured cerebellar granule neurons revealed that the primary cilium dynamically moves with the basal body (Trivedi et al., 2014). To study the subcellular localization of the primary cilium during saltatory movement in chain-forming neuroblasts, Arl13b::Venus was introduced into V-SVZ cells dissected from P0-P1 GFP::Cent2 mice (Fig. 2A). Saltatory movement of neuroblasts was divided into three phases: leadingprocess extension phase, swelling-formation phase, and somal translocation phase, as reported previously (Sawada et al., 2018). Three-dimensional confocal time-lapse imaging revealed that the primary cilium is submerged in the cytosol or extended from the cell surface (Fig. $2 B-E^{\prime}$ ). The proportion of primary cilium extension from the cell surface was significantly higher in swellingformation phase than in other phases (Fig. $2 C-F$ ), suggesting that the extension of the primary cilium is promoted transiently before somal translocation in cultured chain-forming neuroblasts.

During neuronal deceleration in the postnatal OB, V-SVZderived neuroblasts transiently form a filopodium-like lateral protrusion (FLP) from the proximal leading process in the swelling-formation phase, which coincides with the pausing of somal translocation (Sawada et al., 2018). We found that both chain-forming and FLP-bearing neuroblasts extend a primary cilium from the cell surface during the swelling-formation phase (Fig. $2 B-E^{\prime}, G-J^{\prime}$ ). The basal body with extended primary cilium was observed to be continuously migrating forward during the swelling-formation phase in chain-forming neuroblasts (Fig. 2B; 10-14 min), but not in FLP-bearing ones (Fig. 2G), suggesting that FLP formation is related to the migration termination of the primary cilium in the leading process.

Together, these results suggest that the primary cilium localization changes spatiotemporally during saltatory movement in cultured V-SVZ-derived migrating neuroblasts.

\section{Inhibition of Kif3A and IFT88 impairs ciliogenesis and saltatory movement of V-SVZ-derived neuroblasts}

The IFT molecules Kif3A and IFT88 control formation of the primary cilium and migration of pyramidal neurons (Chen et al., 2019) and interneurons (Baudoin et al., 2012) in the embryonic cerebral cortex. Loss of function of Kif3a or Ift 88 causes impaired ciliogenesis, leading process morphology, and centrosomal movement in the MGE-derived cortical interneurons, leading to their abnormal directionality during migration and altered final distribution in the cerebral cortex (Baudoin et al., 2012). To examine the effects of Kif3A inhibition on V-SVZ-derived neuroblasts in the RMS, we injected a lentivirus expressing a dominant-negative form of Kif3A (dnKif3A) and Venus into the $\mathrm{V}-\mathrm{SVZ}$ of adult WT mice. At $7 \mathrm{~d}$ postinjection (dpi), the proportion of neuroblasts with an Arl13b ${ }^{+}$primary cilium (Fig. $3 A-C$ ) and of those that had reached the OB (Fig. 3D) was decreased by dnKif3A expression, suggesting that ciliogenesis and migration toward the OB were disturbed. Unlike the deformation of leading process reported in Kif3A-deficient MGE-derived interneurons (Baudoin et al., 2012), the morphology of dnKif3A-expressing chain-forming neuroblasts was indistinguishable from the control (Fig. $3 A, B$ ). In the RMS, the proportion of neuroblasts pointing toward the $\mathrm{OB}$ was significantly decreased by dnKif3A expression (Fig. 3E). Similar defects in ciliogenesis and rostral migration in the $\mathrm{V}$-SVZ-derived neuroblasts were also observed by IFT88-KD (Fig. 3F-H). At $14 \mathrm{dpi}$, a significantly larger proportion of V-SVZ-derived neuroblasts was observed to remain in the RMS in the dnKif3A-expressing group compared with the 

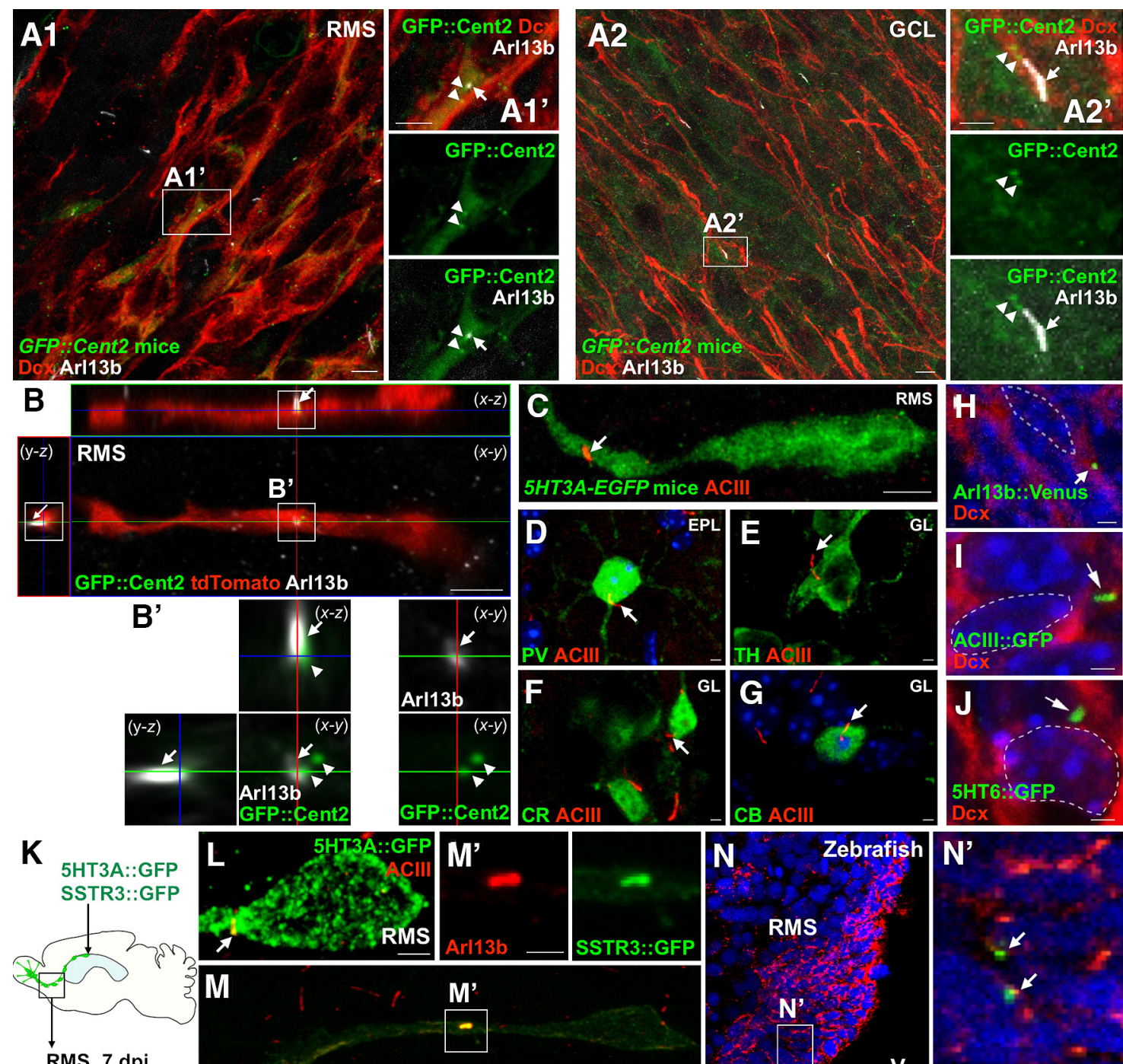

RMS, 7 dpi

(L, M, M')
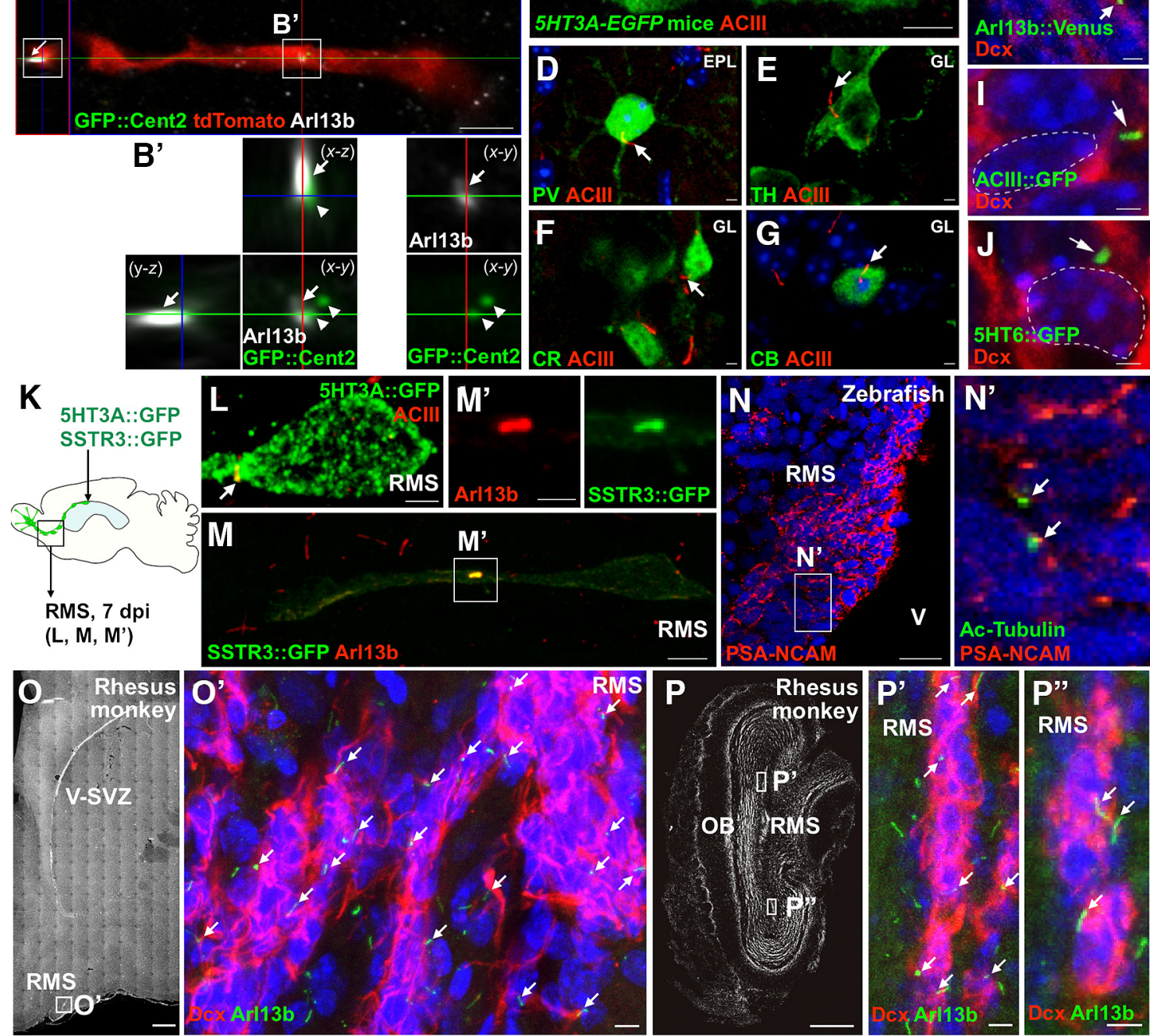

\section{SSTR3::GFP Arl13b}

RMS
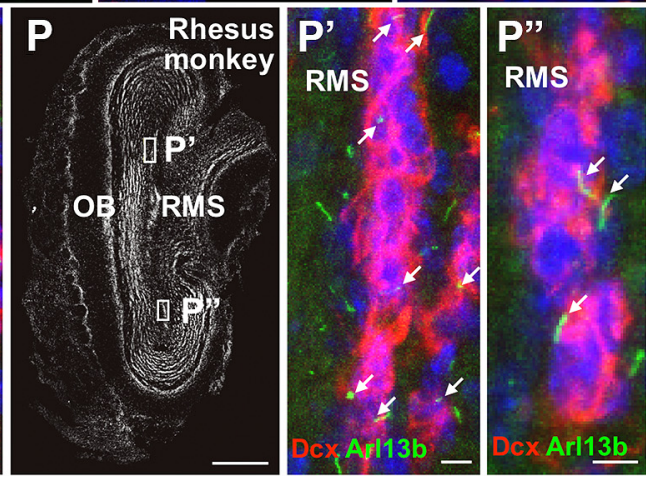

Figure 1. Expression of ciliary markers in migrating neuroblasts in the RMS of the postnatal vertebrate brain. $\boldsymbol{A}-\boldsymbol{A 2} \mathbf{2}^{\prime}$, Representative images of $\mathrm{RMS}\left(\boldsymbol{A 1}, \boldsymbol{A} \mathbf{1}^{\prime}\right)$ and $0 B\left(\boldsymbol{A 2}, \boldsymbol{A 2} \mathbf{2}^{\prime}\right)$ sections of adult GFP::Cent2 mice stained for GFP (green), Dcx (red), and Arl13b (white). $\boldsymbol{A} \mathbf{1}, \mathbf{A 2}$, Boxed areas are enlarged in $\boldsymbol{A} \boldsymbol{1}^{\prime}$ and $\boldsymbol{A 2}^{\prime}$, respectively. Dcx ${ }^{+}$neuroblasts have Arl13b ${ }^{+}$structures (arrows) close to one of the pair of GFP::Cent2 ${ }^{+}$centrioles (arrowheads) in the RMS $\left(\boldsymbol{A} \mathbf{1}^{\prime}\right)$ and OB $\left(\boldsymbol{A 2 ^ { \prime }}\right) \cdot \boldsymbol{B}, \boldsymbol{B}^{\prime}$, Representative image of tdTomato- and GFP::Cent2-labeled cells in the RMS. Lentivirus expressing GFP::Cent2 and Cre was injected into the V-SVZ of adult R26-tdTomato mice. A sagittal section of the RMS of R26-tdTomato mice at 6 dpi stained for GFP (green), DsRed (red), and Arl13b (white, arrows) is shown. $\boldsymbol{B}$, Boxed areas are enlarged in $\boldsymbol{B}^{\prime} \cdot \boldsymbol{B}^{\prime}$, An Arl13b ${ }^{+}$structure (arrows) is located close to one of the pair of GFP::Cent2 ${ }^{+}$centrioles (arrowheads). $\boldsymbol{C}$, Representative image of an RMS section in 5HT3A-EGFP mice (Inta et al., 2008) stained for GFP (green) and ACIII (red). Arrow indicates an ACIII ${ }^{+}$structure. D- $\mathbf{G}$, Representative images of 0 B sections in WT mice stained for olfactory interneuron markers (green; $\boldsymbol{D}, \mathrm{PV} ; \boldsymbol{E}, \mathrm{TH} ; \boldsymbol{F}$, calretinin $[\mathrm{CR}] ; \boldsymbol{G}, \mathrm{CB}$ ) and ACIII (red). Arrows indicate ACIII ${ }^{+}$structures in the olfactory interneurons. EPL, External plexiform layer; $\mathrm{GL}, \mathrm{glomerular} \mathrm{layer.}$ $\boldsymbol{H}-\boldsymbol{J}$, Localization of fluorescent protein-fused ciliary markers in migrating neuroblasts in the adult RMS. Arl13b::Venus $(\boldsymbol{H})$, ACIII::GFP (I), or 5HT6::GFP $(\boldsymbol{J})$ accumulated as spots (arrows) in the perinuclear region of $\mathrm{Dcx}{ }^{+}$neuroblasts. Dashed lines indicate the boundaries of individual nuclei. Blue represents Hoechst 33342-stained nuclei. $\boldsymbol{K}-\boldsymbol{M}^{\prime}$, Colocalization of GFP-fused 5HT3A and SSTR3 with ciliary markers in migrating neuroblasts in the adult RMS. Retroviruses expressing 5HT3A::GFP or neuroblasts expressing SSTR3::GFP were injected into the V-SVZ of adult WT mice (K). 5HT3A::GFP signal $\left(\boldsymbol{L}\right.$, green) is observed in the ACIII ${ }^{+}$structure ( $L$, arrow) in the adult RMS. SSTR3::GFP signal $\left(M, M^{\prime}\right.$, green) is localized in the Arl13b ${ }^{+}$structure $\left(M, M^{\prime}\right.$, red) in the leading process of migrating neuroblasts in the RMS. $\boldsymbol{M}$, Boxed area is enlarged in $\boldsymbol{M}^{\prime}$. $\mathbf{N}-\boldsymbol{N}^{\prime}$, Representative images of a coronal RMS section of an adult zebrafish stained for (Figure legend continues.) 


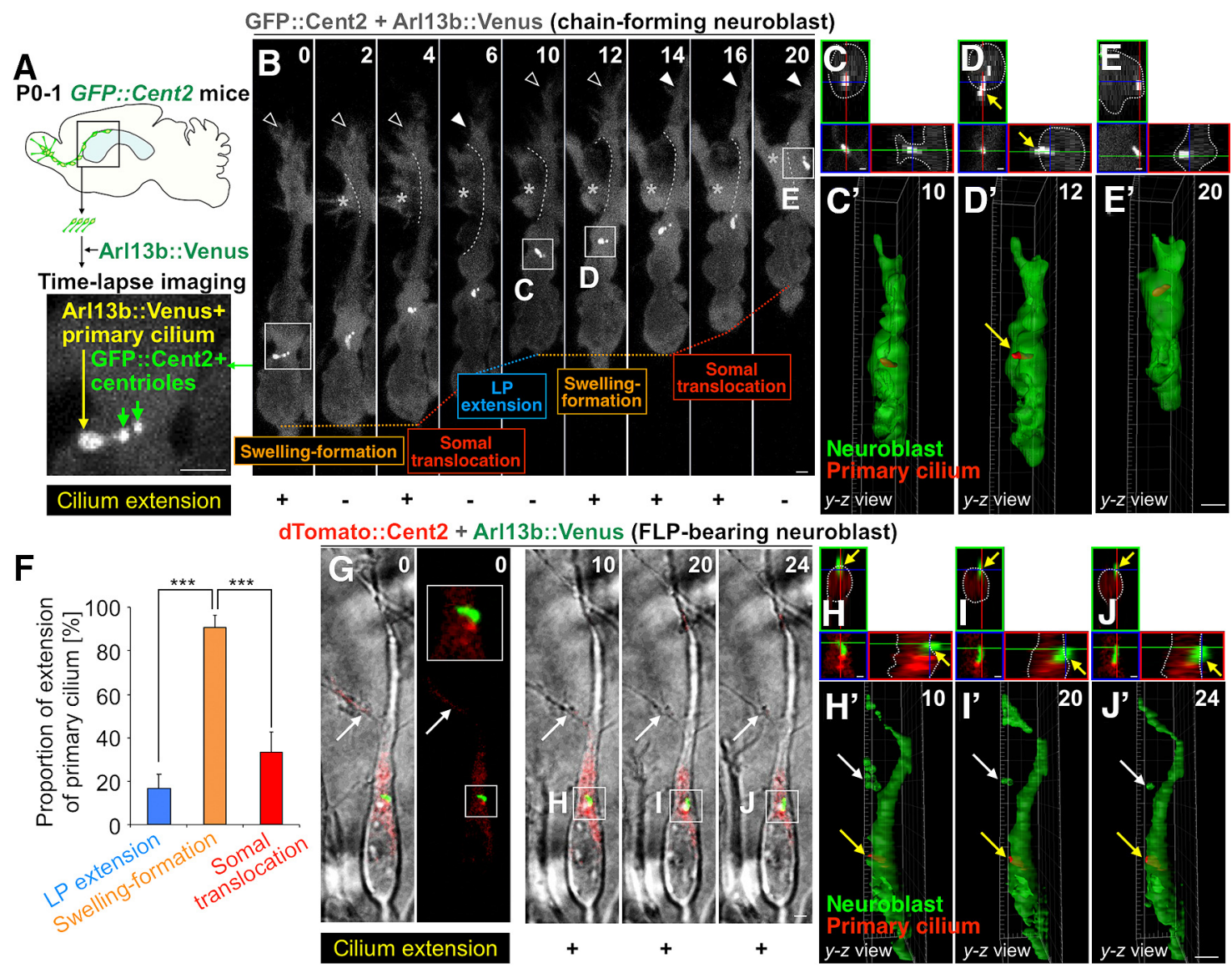

Figure 2. Primary cilium dynamics during saltatory movement of V-SVZ-derived cultured neuroblasts. $A$, Labeling of primary cilia and basal body in V-SVZ-derived cultured neuroblasts. Arl13b::Venus was introduced into V-SVZ cells dissected from P0-P1 GFP:::Cent2 mice. Two GFP::Cent2 ${ }^{+}$centrioles (green arrows) and an Arl13b::Venus ${ }^{+}$primary cilium (yellow arrow) are visualized. $\boldsymbol{B}-\boldsymbol{E}^{\prime}$, Time-lapse imaging of a cultured migrating neuroblast expressing GFP::Cent2 and Arl13b::Venus. $\boldsymbol{B}$, Extension or nonextension of an Arl13b::Venus ${ }^{+}$primary cilium from the cell surface is indicated below by plus and minus signs, respectively. $\boldsymbol{B}$, Boxed areas $(10,12$, and $20 \mathrm{~min})$ are shown orthogonally in $\boldsymbol{C}-\boldsymbol{E}$. $\boldsymbol{B}$, Boxed area ( 0 min) is enlarged in $\boldsymbol{A}$. $\boldsymbol{B}$, Asterisks and dotted lines indicate leading process of a neighboring neuroblast and boundary between two neuroblasts, respectively. Three-dimensional reconstructions $(y-z$ view) of the neuroblast in $\boldsymbol{B}(10,12$, and 20 $\mathrm{min}$ ) are shown in $\boldsymbol{C}^{\prime}-\boldsymbol{E}^{\prime} . \boldsymbol{D}, \boldsymbol{D}^{\prime}$, Yellow arrows indicate an extended primary cilium. $\boldsymbol{B}$, Opened and filled arrowheads indicate spread and collapsed growth cones, respectively. $\boldsymbol{F}$, Proportion of extension of a primary cilium from the cell surface during leading-process (LP) extension phase, swelling-formation phase, and somal translocation phase $\left(\chi_{(2)}^{2}=30.679, p=0.00000022\right.$, Friedman's test; LP extension phase vs swelling-formation phase, $p=0.00041$, LP extension phase vs somal translocation phase, $p=0.0017$, Wilcoxon signed rank test, adjusted with Bonferroni correction; $n=20$ cells from 11 independent cultures prepared on different days). $\mathbf{G}-J^{\prime}$, Time-lapse imaging of a cultured FLP-bearing neuroblast expressing dTomato::Cent2 and Arl13b::Venus. G, Extension of an Arl13b::Venus ${ }^{+}$primary cilium from the cell surface is indicated by a plus sign. Bottom, Boxed area $(0 \mathrm{~min})$ is magnified above to visualize the Arl13b::Venus ${ }^{+}$primary cilium and dTomato::Cent2 ${ }^{+}$basal body. $\mathbf{G}$, White arrows indicate an FLP. Boxed areas $(10,20$, and $24 \mathrm{~min}$ ) are shown orthogonally in $\boldsymbol{H}$-J. Three-dimensional reconstructions (y-z view) of the neuroblast in $\mathbf{G}(10,20$, and $24 \mathrm{~min})$ are shown. $\boldsymbol{H}^{\prime}-\boldsymbol{J}^{\prime}$, Yellow and white arrows indicate an extended primary cilium and FLP, respectively. Scale bars: $A, B, G, 2 \mu \mathrm{m} ; \boldsymbol{C}-\boldsymbol{E}, \boldsymbol{H}-\boldsymbol{J}, 1 \mu \mathrm{m} ; \boldsymbol{C}^{\prime}, \boldsymbol{D}^{\prime}, \boldsymbol{E}^{\prime}, \boldsymbol{H}^{\prime}, \boldsymbol{I}^{\prime}, \boldsymbol{J}^{\prime}, 5 \mu \mathrm{m}$. ${ }^{* * *} p<0.005$. Error bars indicate mean \pm SEM.

control (Fig. 3I). Thus, loss of function of IFT molecules disrupted primary cilium formation and rostral migration in V-SVZ-derived neuroblasts in the RMS.

To study the effect of dnKif3A expression on neuronal saltatory movement in greater detail, we analyzed the migratory behavior of dnKif3A-expressing cultured neuroblasts dissected from the V-SVZ of P0-P1 GFP::Cent2 mice (Fig. 3J-V; Movie 1). The dnKif3A-expressing neuroblasts spent longer in both the resting and migratory phases and showed a decreased migration

(Figure legend continued.) PSA-NCAM (N, $\boldsymbol{N}^{\prime}$, red) and acetylated tubulin ( $\boldsymbol{N}^{\prime}$, Ac-tubulin, green). $\boldsymbol{N}$, Boxed area is enlarged in $\boldsymbol{N}^{\prime}$. Ac-tubulin ${ }^{+}$dots ( $\boldsymbol{N}^{\prime}$, green, arrows) were observed in the PSA-NCAM ${ }^{+}$immature neuroblasts in the adult zebrafish RMS. V, Ventricle. $\mathbf{0}-\boldsymbol{P}^{\prime \prime}$, Representative images of coronal RMS $(\boldsymbol{O}, \mathrm{DAPI})$ and $\mathrm{OB}(\boldsymbol{P}, \mathrm{DAPI})$ sections of an adult rhesus monkey stained for $\mathbf{D c x}\left(\mathbf{O}^{\prime}, \mathbf{P}^{\prime}, \mathbf{P}^{\prime \prime}\right.$, red) and Arl13b $\left(\mathbf{O}^{\prime}, \mathbf{P}^{\prime}, \mathbf{P}^{\prime \prime}\right.$, green). Arl13b ${ }^{+}$structures $\left(\mathbf{O}^{\prime}, \mathbf{P}^{\prime}, \mathbf{P}^{\prime \prime}\right.$, arrows) were observed in the $D c x^{+}$immature neuroblasts in the adult rhesus monkey RMS. Scale bars: $\boldsymbol{A} \mathbf{1}, \boldsymbol{A 2}, \boldsymbol{B}, \boldsymbol{C}, \boldsymbol{M}, \mathbf{O}^{\prime}, \boldsymbol{P}^{\prime}, \boldsymbol{P}^{\prime \prime}, 5 \mu \mathrm{m} ; \boldsymbol{A \mathbf { 1 } ^ { \prime }}, \boldsymbol{A \mathbf { 2 } ^ { \prime }}, \boldsymbol{D}-\boldsymbol{J}, \boldsymbol{L}, \boldsymbol{J}, \boldsymbol{M}^{\prime}, 2 \mu \mathrm{m} ; \boldsymbol{N}, 50 \mu \mathrm{m} ; \boldsymbol{P}, 500$ $\mu \mathrm{m} ; 0,1 \mathrm{~mm}$. speed (Fig. 3J-N). In the resting phase, while dnKif3A expression did not affect leading process extension (Fig. 3O,P), it slowed basal body speed (Fig. 3Q) without affecting its stride (Fig. 3R). Moreover, dnKif3A expression increased the frequency of backward basal body migration in the leading process (Fig. $3 K ; 0-24$ min; Fig. $3 S$ ). In the migratory phase, dnKif3A expression decreased somal speed (Fig. $3 T$ ) but not stride (Fig. $3 U$ ). dnKif3A expression increased the frequency of reversal turn of neuroblasts (Fig. 3K, 24-54 min; Fig. 3V). Similar to MGE-derived interneurons (Baudoin et al., 2012), the phenotypes caused by the inhibition of IFT molecules suggest that the basal body and the primary cilium are involved in the saltatory migration of $\mathrm{V}$-SVZ-derived neuroblasts.

Fine morphology of the primary cilium in migrating neuroblasts in the postnatal RMS

We investigated the ultrastructure of the primary cilium in migrating neuroblasts in the adult RMS using serial-section TEM (36 cells from 5 mice). Immature neuroblasts with migratory 

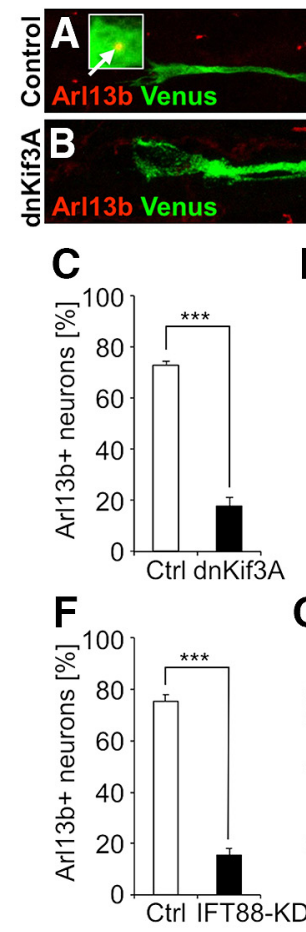

E

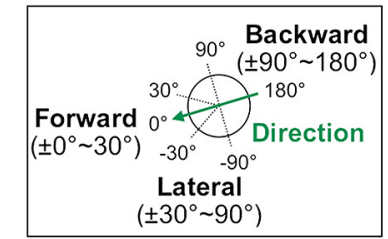

D Distribution

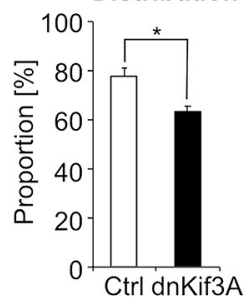

G

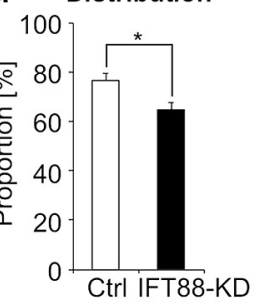

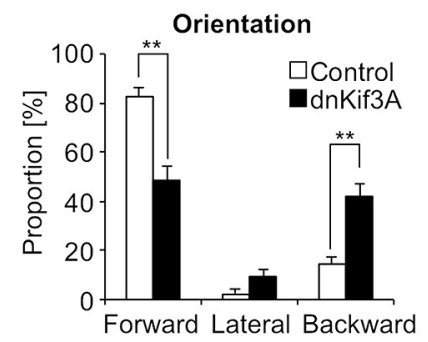

H

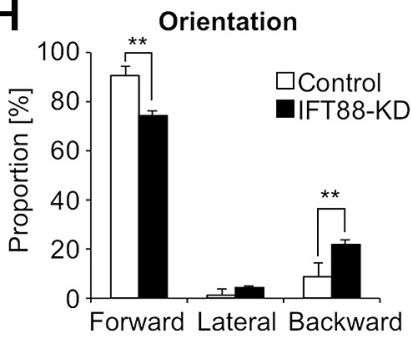

I

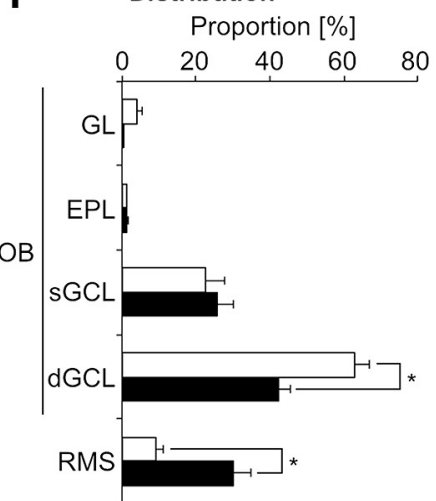

$\square$ Control
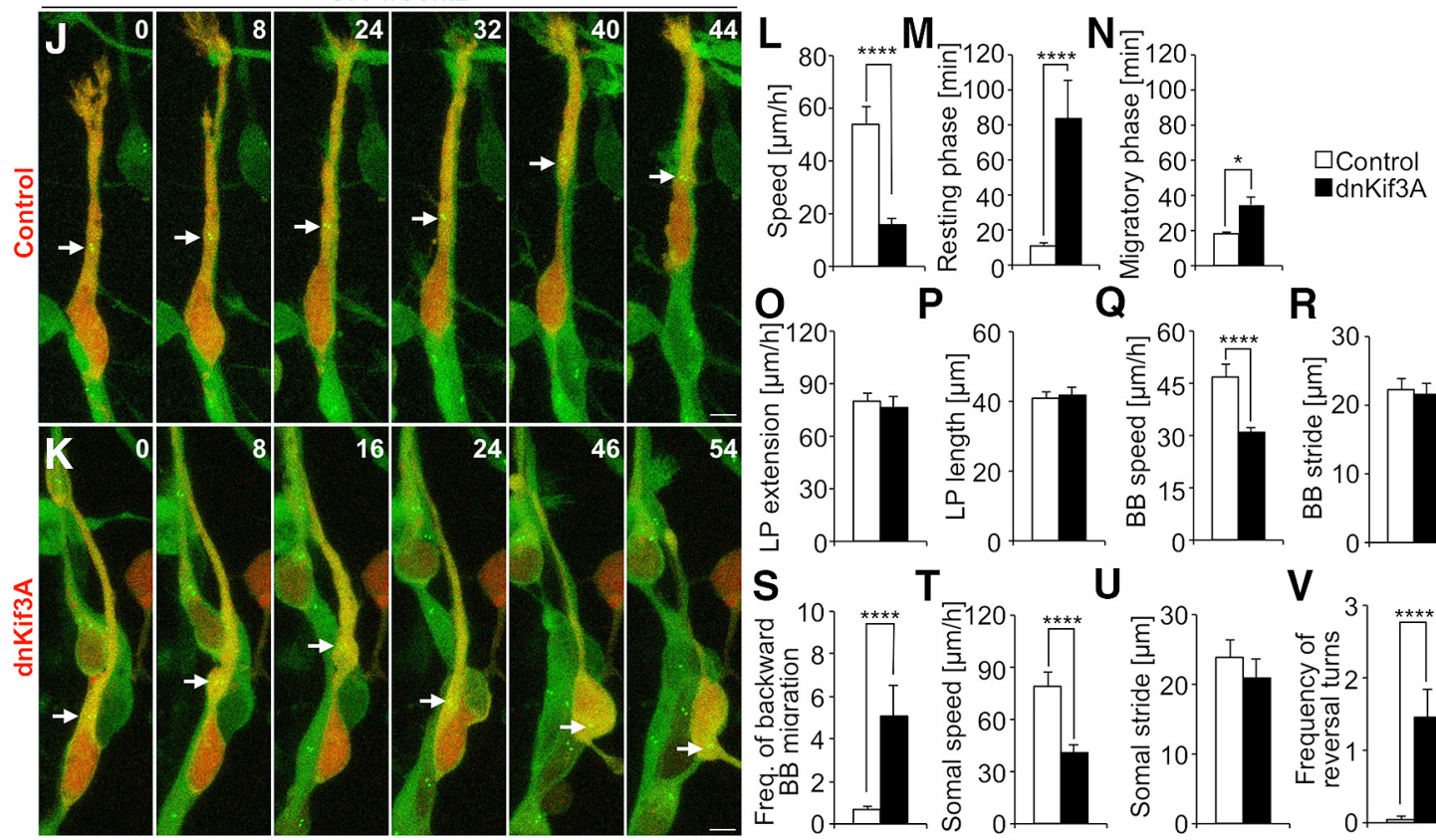

$\mathbf{R}$

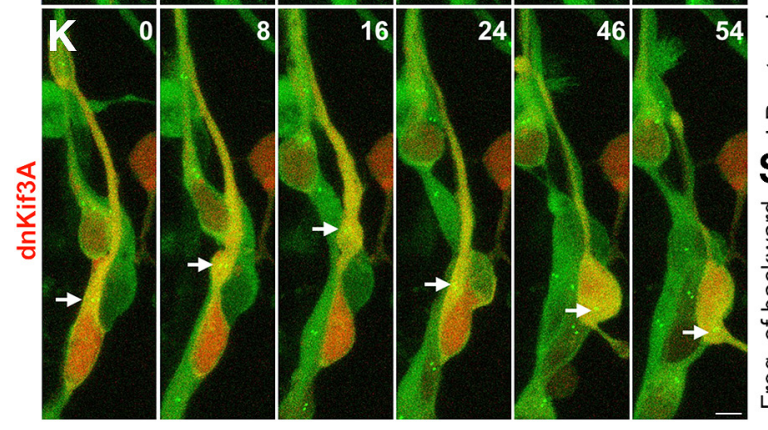

Figure 3. Defects in ciliogenesis and saltatory movement of V-SVZ-derived migrating neuroblasts caused by expressing a dominant-negative form of Kif3A or IFT88 shRNA. A-I, Defects in ciliogenesis, orientation, and final positioning of V-SVZ-derived migrating neuroblasts caused by expressing a dominant-negative form of Kif3A (dnKif3A) (A-E,I) or IFT88 shRNA $(\boldsymbol{F}-\boldsymbol{H})$ in the adult RMS. Lentivirus encoding dnKif3A and Venus $(\boldsymbol{A}-\boldsymbol{E}, \boldsymbol{I})$, or retrovirus encoding IFT88 shRNA and DsRed $(\boldsymbol{F}-\boldsymbol{H})$, was injected into the V-SVZ of WT mice. $\boldsymbol{A}, \boldsymbol{B}$, Representative images of control $(\boldsymbol{A}$, green) and dnKif3A-expressing ( $\boldsymbol{B}$, green) cells in the adult RMS. An Arl13b ${ }^{+}$primary cilium (red, arrow in the enlarged box in $\boldsymbol{A}$ ) was observed in the control but not the dnKif3A-expressing migrating neuroblasts. $C$, Proportion of Arl13b ${ }^{+}$primary cilium-bearing neuroblasts in the control and dnKif3A-expressing groups at $7 \mathrm{dpi}\left(t_{(4)}=15.76, p=9.5 \times 10^{-5}\right.$, unpaired $t$ test; $n=3$ mice each). $D$, Distribution of labeled neuroblasts in the $\mathrm{OB}$ of control and dnKif3A-expressing groups at $7 \mathrm{dpi}\left(t_{(6)}=3.62, p=0.011\right.$, unpaired $t$ test; $n=4$ mice each). $\boldsymbol{E}$, Orientation of labeled neuroblasts in the control and dnKif3A-expressing groups at $7 \mathrm{dpi}\left(F_{\text {group }(1,6)}=0.0003, p_{\text {group }}=0.99, F_{\text {orientation }(2,12)}=79.8, p_{\text {orientation }}=1.2 \times 10^{-7}, F_{\text {group } \times \text { orientation }(2,12)}=21.8, p_{\text {group } \times \text { orientation }}=\right.$ 0.00010 , two-way repeated-measures ANOVA; control vs dnKif3A, $t_{(6)}=5.0, p=0.0074$ in forward orientation; $t_{(6)}=-4.89, p=0.0082$ in backward orientation; unpaired $t$ test; $n=4$ mice each). $\boldsymbol{F}$, Proportion of Arl13b ${ }^{+}$primary cilium-bearing neuroblasts in the control and IFT88-KD groups at $7 \mathrm{dpi}\left(t_{(4)}=17.3, p=6.6 \times 10^{-5}\right.$, unpaired $t$ test; $n=3$ mice each). $\mathbf{G}$, Distribution of labeled neuroblasts in the $\mathrm{OB}$ of control and IFT88-KD groups at $7 \mathrm{dpi}\left(t_{(5)}=2.92, p=0.033\right.$, unpaired $t$ test; control, $n=3$ mice; IFT88-KD, $n=4$ mice). $\boldsymbol{H}$, Orientation of labeled neuroblasts in the control and IFT88-KD groups at $7 \mathrm{dpi}\left(F_{\text {group }(1,5)}=0.056, p_{\text {group }}=0.82, F_{\text {orientation }(2,10)}=1101.8, p_{\text {orientation }}=1.9 \times 10^{-12}, F_{\text {group } \times \text { orientation }(2,10)}=34.0, p_{\text {group } \times \text { orientation }}=3.5 \times 10^{-5}\right.$, two-way repeated-measures ANOVA; control vs IFT88-KD, $t_{(5)}=6.0, p=0.0054$ in forward orientation; $t_{(5)}=-5.86, p=0.0061$ in backward orientation; unpaired $t$ test; control, $n=3$ mice; IFT88-KD, $n=4$ mice). $I$, The final positioning of labeled neuroblasts in the $0 \mathrm{~B}$ and RMS of control and dnKif3A-expressing groups at 14 dpi $\left(F_{\text {group }(1,6)}=-0.0001, p_{\text {group }}=1, F_{\text {layer }(4,24)}=69.3\right.$, $p_{\text {layer }}=7.8 \times 10^{-13}, F_{\text {group } \times \text { layer }(4,24)}=8.82, p_{\text {group } \times \text { layer }}=0.00016$, two-way repeated-measures ANOVA; control vs dnKif3A, $t_{(6)}=-4.09, p=0.032$ in RMS; $t_{(6)}=4.05, p=0.034$ in dGCL; unpaired $t$ test; $n=4$ mice each). $\boldsymbol{J}, \boldsymbol{K}$, Time-lapse images of control $(\boldsymbol{J}$, red) and dnKif3A-expressing $(\boldsymbol{K}$, red $)$ cultured neuroblasts (green) derived from the V-SVZ of GFP::Cent2 mice. Arrows indicate GFP::Cent2 ${ }^{+}$centrioles. $\boldsymbol{L}-\boldsymbol{V}$, Migration speed $\left(\boldsymbol{L} ; p=5.3 \times 10^{-6}\right.$, Welch's $t$ test), durations of resting $\left(\boldsymbol{M} ; \mathbf{W}=28, p=3.5 \times 10^{-7}\right.$, Mann-Whitney $\boldsymbol{U}$ test), and (Figure legend continues.) 


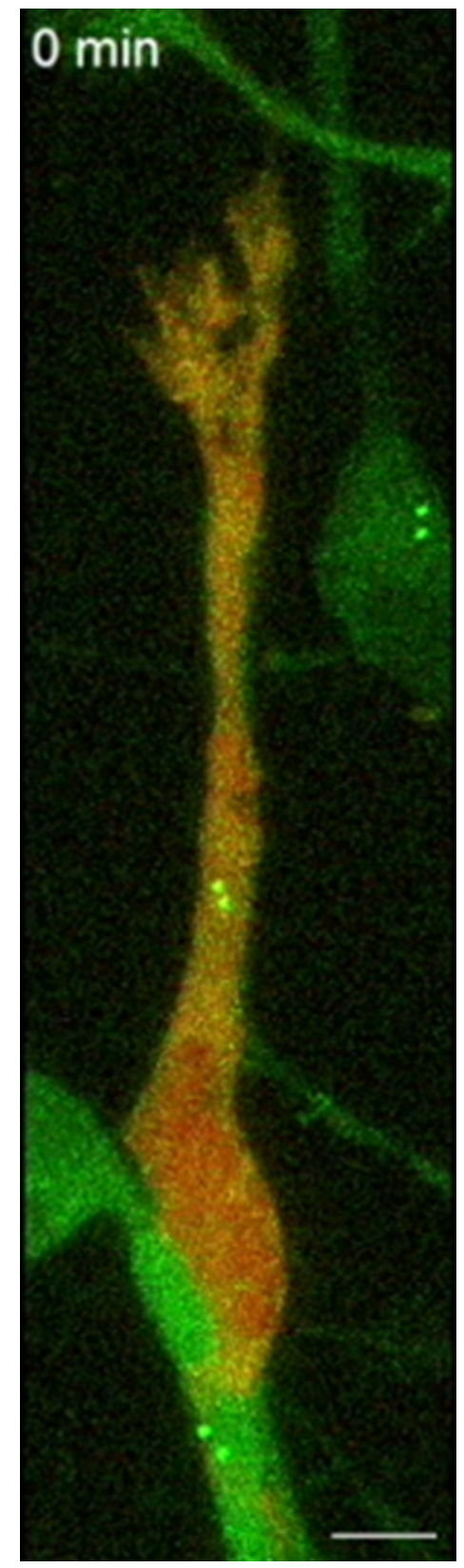

Movie 1. Time-lapse imaging of control (red) and dnKif3Aexpressing (red) neuroblasts (green) derived from the V-SVZ of GFP.: cent2 mice. features in the RMS were identified based on their electron-dense nucleus with multiple nucleoli, dark cytoplasm with many free ribosomes, and the presence of extracellular spaces between neuroblasts, as described previously (Doetsch et al., 1997). Serial TEM images of migratory neuroblasts in the RMS showed either a pair of centrioles (Fig. 4A; 5 of 36 cells), or a basal body with an immature or mature primary cilium (Fig. $4 B-E^{\prime} ; 31$ of 36 cells). MTs were radially extended from the basal body of the primary cilium (Fig. $4 D ; z=1$ and 2), suggesting that the basal body acts as a MT-organizing center in migrating neuroblasts. The immature or mature primary cilium attached to the basal body (31 cells) was further classified into four types: ciliary vesicle, procilium, nonextended primary cilium, and extended primary cilium (Fig. 4B-E; see below).

In previous studies on primary cilium development in immature neurons, two types of immature forms of the primary cilium have been described: ciliary vesicles (Baudoin et al., 2012) and procilium (Arellano et al., 2012). Ciliary vesicles are attached to the basal body in the cytosol (Baudoin et al., 2012). The procilium is a membrane protrusion from the plasma membrane containing no axonemal structures (Arellano et al., 2012). We found immature primary cilia similar to ciliary vesicles (Fig. $4 B ; 6$ of 31 cells) and to the procilium (Fig. $4 C$; 4 of 31 cells) in migratory neuroblasts in the RMS. In addition, elongated ciliary structures with a $9+0$ ciliary axoneme and a ciliary pocket, a typical morphology of the mature primary cilium (Sorokin, 1962), were observed in migratory neuroblasts in the RMS (Fig. 4D-F; 21 of 31 cells). The tip of the primary cilium of these cells (21 cells) was observed to be either nonextended (Fig. $4 D, D^{\prime}$; 18 cells) or extended (Fig. $4 E, E^{\prime} ; 3$ cells) from the cell surface, which we defined as a "nonextended primary cilium" and an "extended primary cilium," respectively. Interestingly, the tip of the nonextended primary cilium appeared to have access to the extracellular environment, even under its submerged state (Fig. $4 D^{\prime}$, yellow arrows). Collectively, these observations suggest that the primary cilium of immature migratory neuroblasts showed diverse morphologies in the postnatal RMS.

\section{Three-dimensional ultrastructural features of migrating neuroblasts in the postnatal RMS analyzed by SBF-SEM}

SBF-SEM is a powerful tool to study both whole cell morphology and its subcellular organs at an ultrastructural level (Briggman and Bock, 2012; Denk et al., 2012; Ohno et al., 2015). Since chain-forming neuroblasts align along the rostrocaudal axis in the anterior RMS, repeated coronal sectioning and image acquisition of RMS tissues by SBF-SEM efficiently include migrating neuroblasts. To establish criteria for celltype identification, ultrastructural features of adult mouse RMS cells observed under SBF-SEM (Figs. 5, 6; Movie 2) were compared with those observed under TEM, which have been reported previously (Doetsch et al., 1997).

Immature migratory neuroblasts could be identified in the SBF-SEM images by a smooth cell contour, dark cytoplasm with small Golgi apparatus, and a few short endoplasmic-reticulum fragments, and the presence of extracellular spaces, similar to the TEM images, as we reported previously (Kaneko et al., 2018; Sawada et al., 2018) (Fig. 5A). While the TEM images clearly showed adherens junction-like structures and an MT network in migratory neuroblasts (Doetsch et al., 1997; Fujikake et al., 2018; Sawada et al., 2018), our SBF-SEM images could not clearly visualize these structures. By performing 3D reconstruction of neuroblasts in the RMS, we examined 330 cells with whole morphology and 88 cells with partial morphology, which lack
(Figure legend continued.) migratory phases ( $\boldsymbol{N} ; p=0.002$, Welch's $t$ test), leading process (LP) extension speed ( $\mathbf{O})$, maximum LP length $(\boldsymbol{P})$, basal body (BB) speed ( $\mathbf{Q} ; \mathrm{W}=6669.5, p=$ $6.82 \times 10^{-6}$, Mann-Whitney $U$ test), BB stride $(\boldsymbol{R})$, frequency of backward BB migration $(\boldsymbol{S}$; $\mathrm{W}=73, p=3.17 \times 10^{-5}$, Mann-Whitney $U$ test $)$, somal speed $(\boldsymbol{T} ; p=0.00012$, Welch's $t$ test), somal stride $(\boldsymbol{U})$, and frequency of polarity reversal $\left(\boldsymbol{V} ; \boldsymbol{W}=93, p=2.9 \times 10^{-5}\right.$, Mann-Whitney $U$ test) in control and dnKif3A-expressing cultured V-SVZ-derived neuroblasts ( $\boldsymbol{L}-\boldsymbol{V}$; control, $n=21$ cells; dnKif3A, $n=24$ cells; five independent cultures prepared on different days). Scale bars: $\boldsymbol{A}, \boldsymbol{B}, \boldsymbol{J}, \boldsymbol{K}, 5 \mu \mathrm{m} .{ }^{*} p<0.05 ;{ }^{* *} p<0.01 ;{ }^{* * *} p<0.005 ;{ }^{* * * *} p<$ 0.001 ; adjusted with Bonferroni correction in $\boldsymbol{E}, \boldsymbol{H}$, and $\boldsymbol{I}$. Error bars indicate mean \pm SEM. 

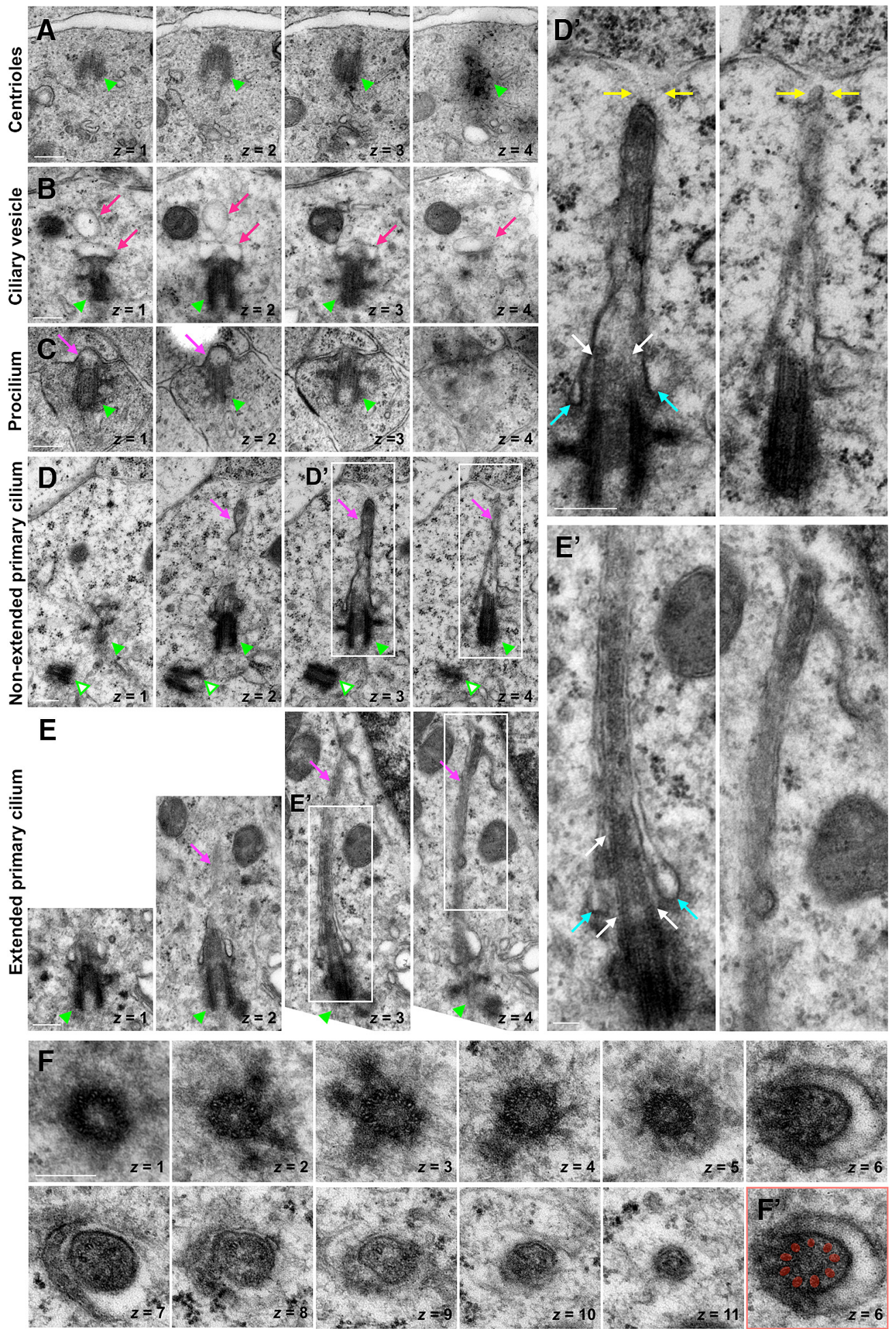

Figure 4. In vivo identification of the primary cilium in migrating neuroblasts in the adult RMS. $\boldsymbol{A}-\boldsymbol{E}^{\prime}, \mathrm{TEM}$ images of centrioles $(\boldsymbol{A})$, ciliary vesicles $(\boldsymbol{B}$, pink arrows), a procilium $(\boldsymbol{C}$, magenta arrow), a nonextended primary cilium ( $\boldsymbol{D}$, magenta arrow), and an extended primary cilium ( $\boldsymbol{E}$, magenta arrow) in neuroblasts in the adult RMS. Green filled arrowheads indicate centrioles $(\boldsymbol{A})$ or mother centrioles $(\boldsymbol{B}-\boldsymbol{E})$. $\boldsymbol{D}$, Green open arrowheads indicate daughter centrioles. $\boldsymbol{D}, \boldsymbol{E}$, Boxed areas are enlarged in $\boldsymbol{D}^{\prime}$ and $\boldsymbol{E}^{\prime}$, respectively. $\boldsymbol{D}^{\prime}, \boldsymbol{E}^{\prime}$, Blue and white arrows indicate ciliary pockets and the ciliary axoneme, respectively. $\boldsymbol{D}^{\prime}$, The tip of the nonextended primary cilium appeared to have access to the extracellular environment (yellow arrows). $\boldsymbol{F}, \boldsymbol{F}^{\prime}$, Sequential coronal TEM images of the primary cilium in neuroblasts in the adult RMS. Coronal images of the basal body $(z=1)$, transition zone $(z=2-5)$, and doublet zone $(z=6-11)$ of the primary cilium are shown. The primary cilium shows $9+0$ axoneme structure $\left(\boldsymbol{F}, z=6 ; \boldsymbol{F}^{\prime}\right.$, red). Scale bars, $200 \mathrm{~nm}$.

part of the leading process and/or soma (for a detailed definition of "partial cells," see Materials and Methods). The majority of identified neuroblasts were oriented rostrally $(87.1 \pm 1.4 \%, 292$ of 330 whole cells and 72 of 88 partial cells from 5 mice) and tightly attached to their neighboring neuroblasts, forming chains in the RMS (Fig. 5A).

In addition to migratory neuroblasts, tunnel-forming astrocytes, oligodendrocytes, and microglia were observed in and 

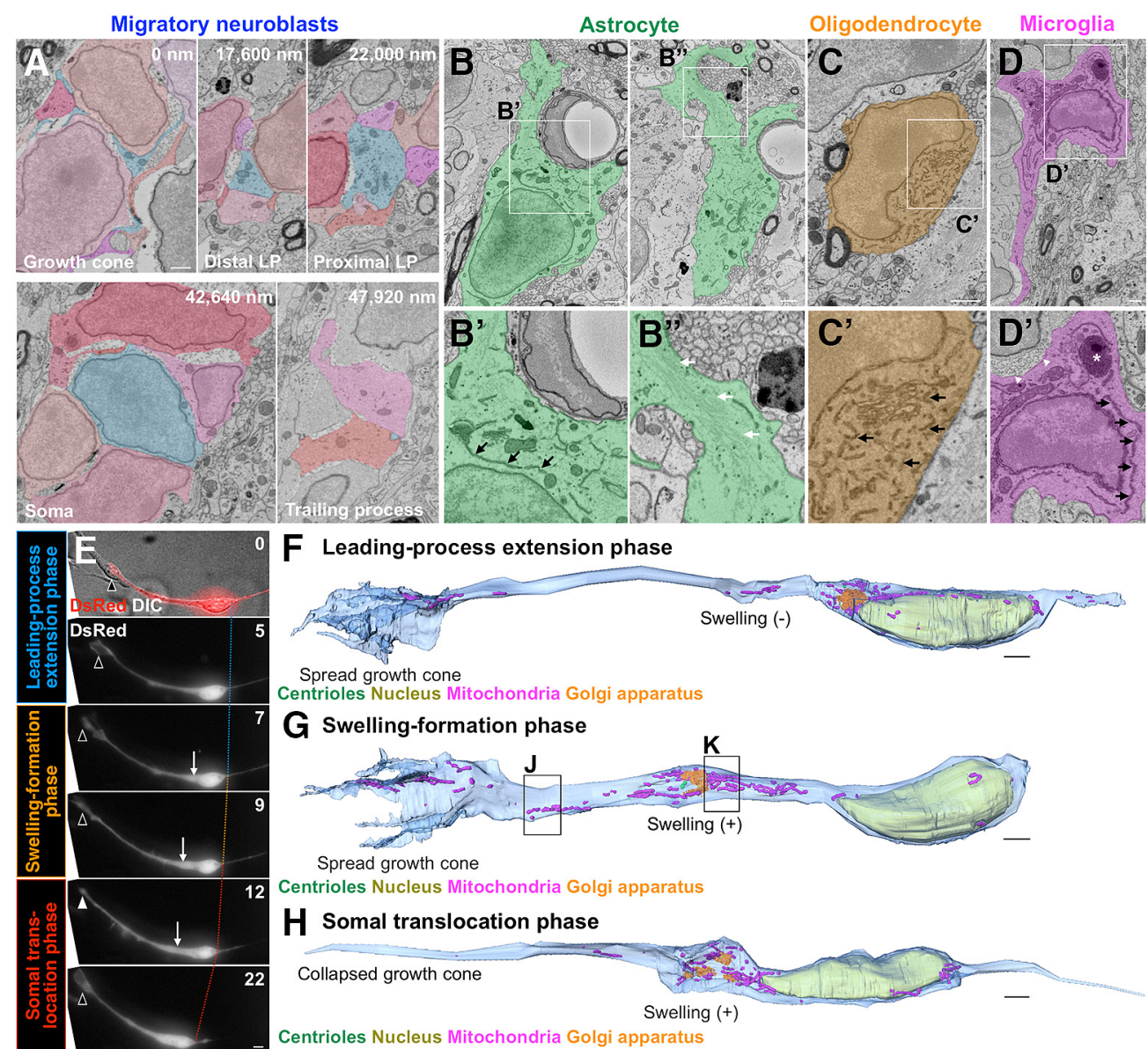

F Leading-process extension phase

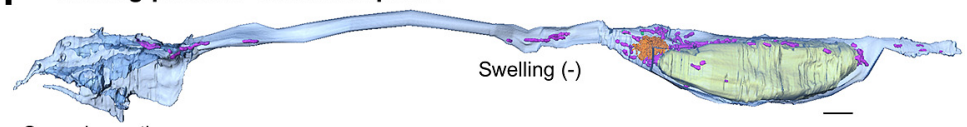

Spread growth cone

Centrioles Nucleus Mitochondria Golgi apparatus

G Swelling-formation phase

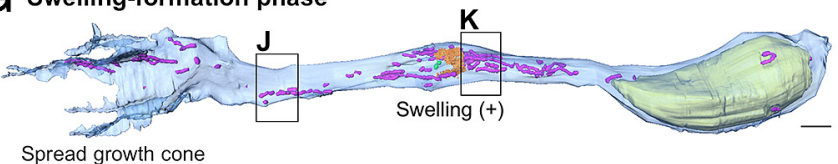

Spread growth cone

Centrioles Nucleus Mitochondria Golgi apparatus

H Somal translocation phase

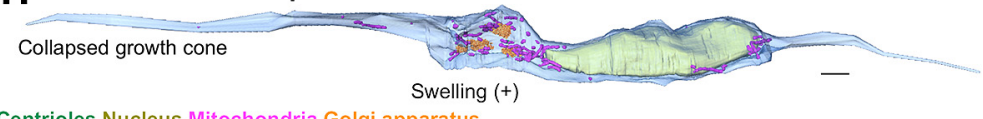

Centrioles Nucleus Mitochondria Golgi apparatus
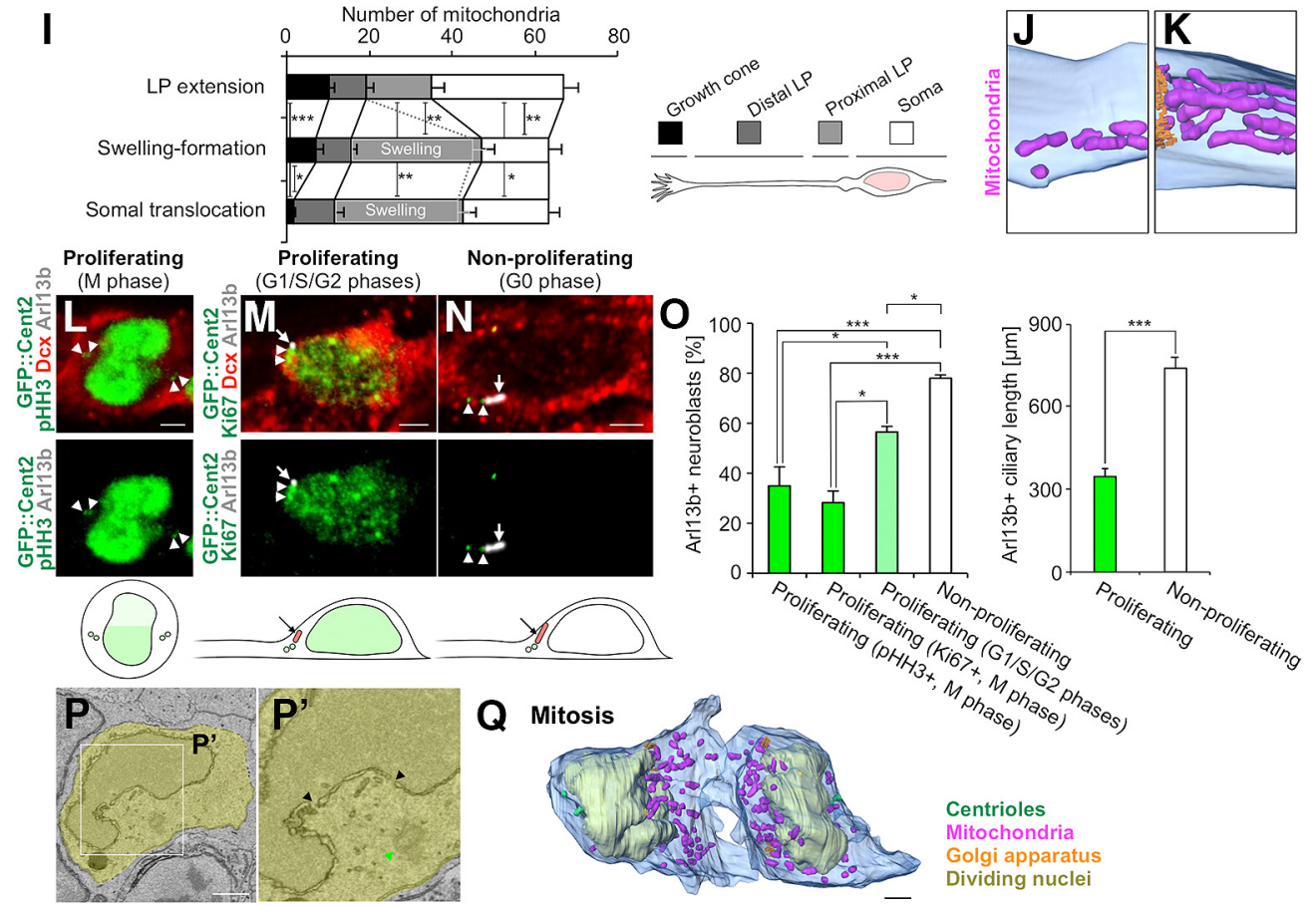

Centrioles

Mitochondria

Golgi apparatus
Dividing nuclei

Figure 5. Ultrastructural features of V-SVZ-derived neuroblasts during proliferation and saltatory movement analyzed by SBF-SEM. A, Representative SBF-SEM images of migratory neuroblasts in the adult RMS. The migratory neuroblast consists of the growth cone, distal/proximal part of the leading process ( $(\mathrm{P})$, soma, and trailing process (blue). Migratory neuroblasts are tightly attached with their neighbors. Red represents all the neighbors. Between neuroblasts, extracellular spaces were also observed. $\boldsymbol{B}-\boldsymbol{D}^{\prime}$, Representative SBF-SEM images of astrocytes $\left(\boldsymbol{B}-\boldsymbol{B}^{\prime \prime}\right)$, oligodendrocytes $\left(\boldsymbol{C}, \boldsymbol{C}^{\prime}\right)$, and microglia $\left(\boldsymbol{D}, \boldsymbol{D}^{\prime}\right)$ in the adult RMS. Astrocytes showed long endoplasmic reticulum $\left(\boldsymbol{B}^{\prime}\right.$, black arrows) and intermediate filaments ( $\boldsymbol{B}^{\prime \prime}$, white arrows), and were tightly attached to a blood vessel $\left(\boldsymbol{B}, \boldsymbol{B}^{\prime}\right)$. Oligodendrocytes showed a round cell contour with short and dilated endoplasmic reticulum ( $\boldsymbol{C}^{\prime}$, back arrows), and direct contacts to myelinated and nonmyelinated axons ( $(\boldsymbol{C})$. Microglia showed an irregular cell contour with long processes, dark cytosol with long endoplasmic reticulum ( $\boldsymbol{D}^{\prime}$, black arrows) and a lysosome ( $\boldsymbol{D}^{\prime}$, asterisk), and extracellular spaces ( $\left(\boldsymbol{D}^{\prime}\right.$, white arrowheads). $\boldsymbol{E}$, Time-lapse images for chain migration of DsRed-expressing cultured neuroblasts. Black and white arrowheads indicate spread and (Figure legend continues.) 
around the RMS. Tunnel-forming astrocytes were identified by an irregular contour, light cytoplasm with long endoplasmic reticulum, and abundant intermediate filaments (Fig. 5B- $B^{\prime \prime}$ ), similar to the TEM images that have been reported previously (Doetsch et al., 1997). These astrocytes enwrapped the blood vessels and/or neuronal chains by their processes to form a glial tube in the RMS. Oligodendrocytes were located around the RMS and could be identified by a smooth and round contour, short and dilated endoplasmic reticulum, and direct contacts with myelinated and nonmyelinated axons (Fig. 5C, $C^{\prime}$ ) (Menn et al., 2006). Microglia were identified by an irregular contour with elongated processes, dark cytoplasm with long endoplasmic reticulum and lysosomes, and the presence of extracellular spaces (Fig. 5D, $D^{\prime}$ ) (Capilla-Gonzalez et al., 2014). Thus, SBF-SEM successfully classified all major cell types in the postnatal RMS based on their ultrastructural features.

$\leftarrow$

(Figure legend continued.) collapsed growth cones, respectively. Collapsed growth cones were only observed during somal translocation. Neuroblasts in the LP extension phase showed a spread growth cone with no swelling in their leading process $(0,5 \mathrm{~min})$. Neuroblasts in the swellingformation phase showed a spread growth cone with swelling in their leading process $(7,9 \mathrm{~min})$. Neuroblasts in the somal translocation phase showed a collapsed growth cone with swelling in their leading process (12 min). Arrows indicate swelling. $\boldsymbol{F}-\boldsymbol{H}$, Representative $3 \mathrm{D}$ constructions of migratory neuroblasts (blue) under LP extension phase $(\boldsymbol{F})$, swelling-formation phase $(\boldsymbol{G})$, and somal translocation phase $(\boldsymbol{H})$. Magenta and orange represent all the mitochondria and Golgi apparatus, respectively. Yellow represents nuclei. $\boldsymbol{F}-\boldsymbol{H}$, Interactive 3 D models of neuroblasts in (without the Golgi apparatus) are shown at https://sketchfab.com/3d-models/399a425539e44087a9ba4a5c3dc5f361 (F), https://sketchfab.com/3d-models/fbaa32c3b1b34fdb9af1569cb3973475 (G), and https:// sketchfab.com/3d-models/ee40d9f934e840d18ba61a653fa2f7fc (H). $\boldsymbol{I}$ - $\boldsymbol{K}$, Distribution and morphology of mitochondria in migrating neuroblasts. The number of mitochondria in $L P$ extension phase, swelling-formation phase, and somal translocation phase $\left(\boldsymbol{I} ;\right.$ soma, $F_{(2,42)}=6.44, p=$ 0.0036 , one-way ANOVA; LP extension phase vs swelling-formation phase, $p=0.0032$, LP extension phase vs somal translocation phase, $p=0.048$, Tukey-Kramer test, proximal leading process, $F_{(2.42)}=7.89, p=0.0012$, one-way ANOVA; LP extension phase vs swellingformation phase, $p=0.0035$, LP extension phase vs somal translocation phase, $p=0.004$, Tukey-Kramer test, growth cone, $\chi_{(2)}^{2}=19.2, p=6.9 \times 10^{-5}$, Kruskal-Wallis test; $L P$ extension phase vs somal translocation phase, $t(\infty)=4.19, p=8.3 \times 10^{-5}$, swellingformation phase vs somal translocation phase, $t(\infty)=2.88, p=1.1 \times 10^{-2}$; Steel-Dwass test; $n=3$ mice). Proximal leading process contains swelling in swelling-formation phase and somal translocation phase $(I)$. Most of the mitochondria in the proximal leading process are located in the swelling in swelling-formation phase $(I, 94.3 \pm 1.6 \%)$ and somal translocation phase $(\boldsymbol{I}, 96.9 \pm 1.5 \%)$. $\boldsymbol{J}, \boldsymbol{K}$, Images are magnified views of boxed areas in $\mathbf{G} . \mathbf{L}-\mathbf{0}$, Arl13b expression in proliferating and nonproliferating $\mathrm{Dcx}{ }^{+}$cells in the adult RMS. Representative images of RMS sections of adult GFP::(Cent2 mice stained for GFP (green, dot-like structures), pHH3 ( $\boldsymbol{L}$ ) or Ki67 ( $M, N)$ (green, nucleus), Arl13b (white), and Dcx (red). $L-N$, Arrows and arrowheads indicate the Arl13 $\mathrm{b}^{+}$primary cilium and centrioles, respectively. Proportions of Arl13b ${ }^{+}$primary cilium-bearing neuroblasts in proliferating and nonproliferating $\mathrm{Dcx}{ }^{+}$neuroblasts are shown in $\mathbf{O}$ (left) $\left(F_{(3,8)}=23.15 p=0.00027\right.$ one-way ANOVA; proliferating in $\mathrm{M}$ phase $\left[\mathrm{pHH} 3^{+}\right.$] vs proliferating in $G_{1} / S / G_{2}$ phases, $p=0.047$, proliferating in $M$ phase $\left[\mathrm{pHH} 3^{+}\right]$vs nonproliferating, $p=0.00086$, proliferating in M phase $\left[\mathrm{Ki} 67^{+}\right]$vs proliferating in $\mathrm{G}_{1} / \mathrm{S} / \mathrm{G}_{2}$ phases, $p=0.012$, proliferating in M phase $\left[\mathrm{Ki} 67^{+}\right]$vs nonproliferating, $p=0.00031$, proliferating in $G_{1} / S / G_{2}$ phases vs nonproliferating, $p=0.047$, Tukey-Kramer test; $n=3$ mice). Arl $13 \mathrm{~b}^{+}$ciliary length in proliferating and nonproliferating neuroblasts is shown in $\mathbf{0}$ (right) (proliferating neuroblasts, $342.1 \pm 25.8 \mathrm{~nm}, n=42$ cells from 3 mice; nonproliferating neuroblasts, $737.4 \pm 40.3 \mu \mathrm{m}, n=90$ cells from 3 mice; $\mathrm{W}=3365.5, p=5.7 \times 10^{-13}$, Mann-Whitney $U$ test). $\mathbf{L}-\boldsymbol{N}$, Bottom, Illustrations of the Arl13b ${ }^{+}$primary cilium (pink, black arrows) in proliferating (green nuclei) and nonproliferating (white nucleus) $D \mathrm{Dx}^{+}$cells. Pairs of green dots represent pairs of centrioles. $\boldsymbol{P}, \boldsymbol{P}^{\prime}$, SBF-SEM images of dividing neuroblasts in the adult RMS. Black and green arrowheads indicate a disrupted nuclear membrane and a centriole without a primary cilium, respectively. $\boldsymbol{P}$, Boxed area is enlarged in $\boldsymbol{P}^{\prime}$. $\mathbf{Q}$, Three-dimensional construction of dividing neuroblasts in the adult RMS. Magenta, orange, and green represent mitochondria, Golgi apparatus, and centrioles, respectively. Yellow represents divided nuclei. An interactive 3D model of dividing neuroblasts (without the Golgi apparatus) is shown at https://sketchfab.com/3d-models/528050289d9143caab1c2b35c7fa1b3c. Raw SBF-SEM images in the adult RMS are shown in Movie 2. Scale bars: $\mathbf{A}-\boldsymbol{D}, \boldsymbol{P}, 1 \mu \mathrm{m} ; \boldsymbol{E}, 5 \mu \mathrm{m} ; \boldsymbol{F}-\boldsymbol{H}, \mathbf{L}-\mathbf{N}, \mathbf{Q}$, $2 \mu \mathrm{m}$. Error bars indicate mean \pm SEM. ${ }^{*} p<0.05,{ }^{* *} p<0.01,{ }^{* * *} p<0.005$.
We next compared the SBF-SEM images of neuroblasts with the time-lapse images of cultured chain-forming neuroblasts during saltatory movement (Fig. $5 E-H$ ). The tip of the leading process showed a spread growth-cone-like structure during leading-process extension and swelling-formation phases (Fig. $5 E$, black arrowheads) that collapsed during somal translocation phase (Fig. 5E, white arrowhead). Swelling was observed after the extension of the leading process and before the ending of somal translocation, as reported previously (Ota et al., 2014; Sawada et al., 2018) (Fig. 5E, arrows). Consistent with these findings, 3D reconstruction of cell contour based on the SBF-SEM images visualized a long leading process, with or without swelling, which has a spread or collapsed growth cone at its tip, in addition to an elongated cell body and trailing process (Fig. $5 \mathrm{~F}-\mathrm{H}$; Movies 3, 4, 5 , and 6). Thus, by analyzing the morphology of the swelling and growth cone, we could identify the migration phase of each neuroblast in SBF-SEM images.

Three-dimensional reconstruction also revealed that mitochondria and the Golgi apparatus were mainly distributed in the proximal leading process or swelling, as reported previously (Bellion et al., 2005; Lin-Hendel et al., 2016) (Fig. 5F-H). While there was no significant difference in the numbers of mitochondria in neuroblasts that showed leading process extension, swelling formation, and somal translocation, their distribution was altered depending on saltatory movement (Lin-Hendel et al., 2016) (Fig. $5 F-H$, magenta, Fig. $5 I$ ). Mitochondria located in the proximal leading process were longer than those in the distal leading process (Fig. 5J,K; distal leading process, $0.58 \pm 0.02 \mu \mathrm{m}[n=166$ from 18 cells]; proximal leading process, $1.04 \pm 0.03 \mu \mathrm{m}[n=$ 417 from 18 cells]; $\mathrm{W}=19,594, p=2.6 \times 10^{-16}$, Mann-Whitney $U$ test). Together, these findings suggest that mitochondria and the Golgi apparatus move in a saltatory movement-dependent manner in migratory neuroblasts in vivo.

In contrast to immature neurons migrating in the developing brain, V-SVZ-derived neuroblasts have remarkable mitotic potential during their migration in the RMS (Ikeda et al., 2010; Ponti et al., 2013). To examine the relationship between mitotic states and ciliogenesis in migrating neuroblasts in the RMS, we classified $\mathrm{Dcx}^{+}$cells into proliferating or nonproliferating neuroblasts based on expression of the cell cycle marker Ki67. Proliferating neuroblasts were further divided into two subgroups: $M$ phase (with a mitotic nucleus) or $G_{1} / S / G_{2}$ phases (Fig. $5 L-N$ ). In proliferating neuroblasts, an Arl13b ${ }^{+}$primary cilium was observed less frequently, and its length was shorter, compared with these features in nonproliferating neuroblasts (Fig. 5O). Among these proliferating neuroblasts, the proportion of cilium-bearing neuroblasts was lower in $M$ phase than in $G_{1} / S / G_{2}$ phases (Fig. $5 \mathrm{O}$ ), suggesting that ciliogenesis is transiently suppressed during mitosis. In the SBF-SEM analysis, dividing neuroblasts in the adult RMS possessed two pairs of centrioles, divided nuclei, no apparent Golgi apparatus (Danilov et al., 2009), and no primary cilium (Fig. 5P, Q; Movie 7). Together, these results suggest that mitotic states affect ciliogenesis in migrating neuroblasts in the postnatal RMS.

\section{Changes in subcellular localization and orientation of the primary cilium during saltatory movement of migrating neuroblasts in the postnatal brain}

To visualize the fine whole-cell morphology of migrating neuroblasts in the adult RMS and primary cilium in vivo, we reconstructed their images three-dimensionally (Figs. 6, 7; 330 whole cells from 5 mice). Consequently, a pair of centrioles (Fig. $6 A-B^{\prime}$; Movie 8) or one of four types of primary cilium (Fig. $6 C-J^{\prime}$; Movies 9, 10, 

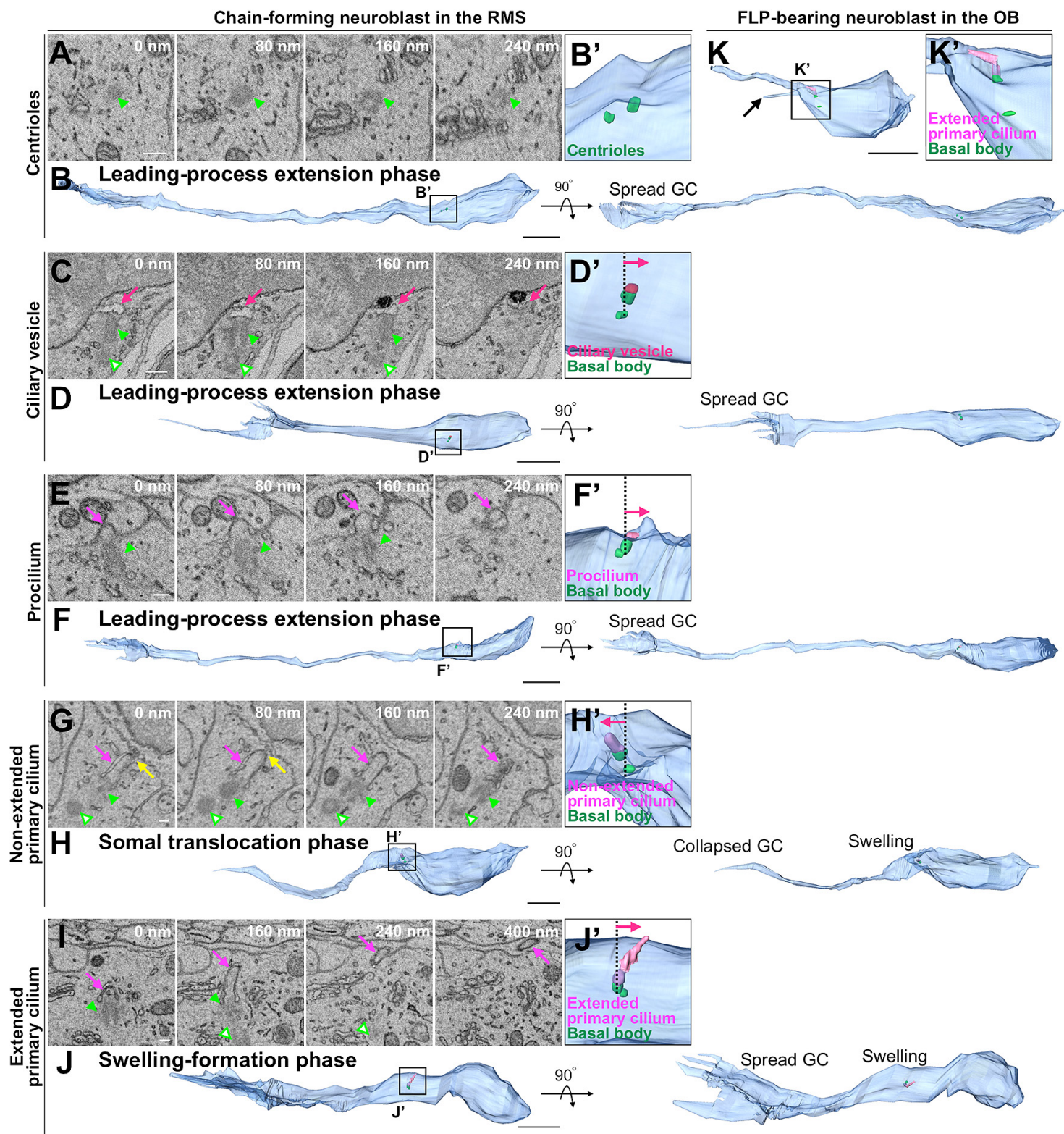

Figure 6. Three-dimensional ultrastructural analysis of the primary cilium in migrating neuroblasts in the adult RMS by SBF-SEM. A-J', Sequential SBF-SEM images and 3D constructions of centrioles $\left(\boldsymbol{A}-\boldsymbol{B}^{\prime}\right)$, a ciliary vesicle $\left(\boldsymbol{C}-\boldsymbol{D}^{\prime}\right.$, pink arrows), a procilium $\left(\boldsymbol{E}-\boldsymbol{F}^{\prime}\right.$, magenta arrows), a nonextended primary cilium $\left(\mathbf{G}-\boldsymbol{H}^{\prime}\right.$, magenta arrows), and an extended primary cilium $\left(\boldsymbol{I}-\boldsymbol{J}^{\prime}\right.$, magenta arrows). Green filled arrowheads indicate centrioles $(\boldsymbol{A})$ or mother centrioles $(\boldsymbol{C}, \boldsymbol{E}, \boldsymbol{G}, \boldsymbol{I})$. Green open arrowheads indicate daughter centrioles $(\boldsymbol{C}, \boldsymbol{G}, \boldsymbol{I}) . \boldsymbol{D}^{\prime}, \boldsymbol{F}^{\prime}, \boldsymbol{H}^{\prime}, \boldsymbol{J}^{\prime}$, Pink arrows indicate primary cilium orientation in the cells. $\boldsymbol{K}, \boldsymbol{K}^{\prime}$, Three-dimensional construction of an extended primary cilium $\left(\boldsymbol{K}^{\prime}\right)$ in an FLP-bearing neuroblast in the adult $0 B$. The arrow indicates an FLP. $\boldsymbol{B}, \boldsymbol{D}, \boldsymbol{F}, \boldsymbol{H}, \boldsymbol{J}, \boldsymbol{K}$, Interactive 3D models of neuroblasts are shown at https://sketchfab.com/3d-models/62e9a69dec414b31a64e8d0158bf7945 (B), https://sketchfab.com/3d-models/1c9eb432686748e8ac537afab59e335a (D), https://sketchfab.com/3d-models/5a5ba2836417475a9bb7513056824055 (F), https://sketchfab.com/3d-models/73abab8f28364a3c99277f7086c19c85 (H), https://sketchfab.com/3dmodels/46cff34b6a9947bfa95fbdd12b0696f4 (J), and https://sketchfab.com/3d-models/0e3963794ca74fc2a15d8cdde4e3c0ee (K). Scale bars: $\boldsymbol{A}, \boldsymbol{C}, \boldsymbol{E}, \boldsymbol{G}, \boldsymbol{I}, 200 \mathrm{~nm} ; \boldsymbol{B}, \boldsymbol{D}, \boldsymbol{F}, \boldsymbol{H}, \boldsymbol{J}, \boldsymbol{K}, 5 \mu \mathrm{m}$.

$11,12)$ was observed adjacent to the Golgi apparatus $(100 \pm$ $0 \%, 330$ whole cells from 5 mice) in migratory neuroblasts.

In the adult RMS, a mature primary cilium was observed in $59.8 \pm 3.3 \%$ of migratory neuroblasts, most of which $(81.8 \pm$ $1.7 \%)$ were classified as the nonextended type (Fig. 7A). The remaining $40.2 \pm 3.3 \%$ had centrioles, ciliary vesicles, or a procilium (Fig. 7A). Consistent with the results of live imaging of cultured neuroblasts (Fig. $2 A-F$ ), the proportions of extended primary cilium and procilium were larger in the swellingformation phase than in other phases of saltatory movement (Fig. $7 B)$. The primary cilium length was not significantly different between nonextended and extended forms in the RMS (Fig. 7C). These results suggest that extension of the mature and immature primary cilium is promoted transiently during the swellingformation phase of saltatory movement in chain-forming migrating neuroblasts in vivo.

While immature neuroblasts migrate in chains at a high speed in the RMS, these neuroblasts detach from chains to migrate individually, and transiently form FLPs during migration termination in the OB (Sawada et al., 2018). In contrast to the chainforming neuroblasts in the RMS, those in the granule cell layer of the OB, which are radially dispersed and have an elongated leading process, could not be included in our imaging volume $(40 \times$ $40 \times 80 \mu \mathrm{m})$. Therefore, we analyzed 25 partial OB cells from 3 mice. We investigated the primary cilium morphology in immature neuroblasts in the $\mathrm{OB}$ and found a mature primary cilium in 


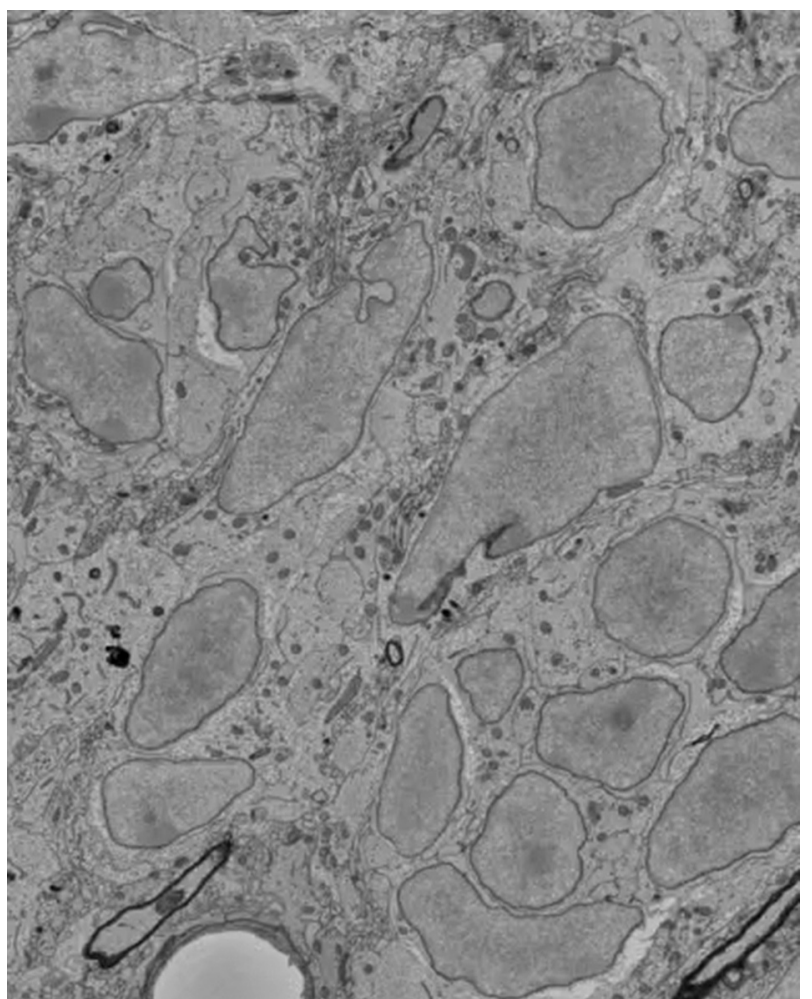

Movie 2. Sequential SBF-SEM images in the adult RMS. Representative sequential SBF-SEM images (1156 sections) in the adult RMS are shown. Neuroblasts shown in Figure 5F, G, H appear in yellow, red, and blue, respectively. Dividing neuroblasts shown in Figure $5 \mathrm{Q}$ appear in green.
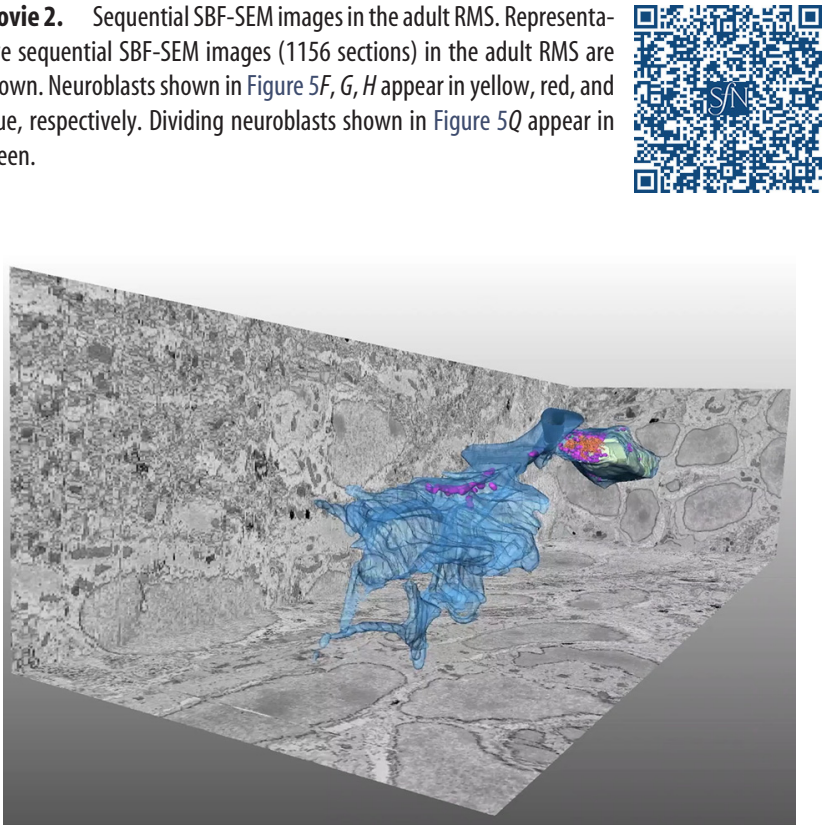

Movie 3. Three-dimensional reconstruction of a migratory neuroblast in the adult RMS. Blue represents the cell contour of the migratory neuroblast. Green, yellow, orange, and magenta represent centrioles, nucleus, Golgi apparatus, and mitochondria, respectively.

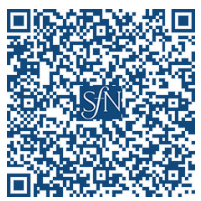

$84.9 \pm 11.4 \%$ of these neuroblasts (Fig. $7 A$ ). The proportion of extended primary cilium was significantly larger in FLP-bearing neuroblasts than in neuroblasts without FLP (Fig. $6 K, K^{\prime}$; FLPbearing neuroblasts, $95.2 \pm 4.8 \%$; neuroblasts without FLP, $22.2 \pm 14.7 \% ; t_{(4)}=-4.73, p=0.0091$, paired $t$ test; $n=3$ mice). Furthermore, the extended primary cilium was longer than the

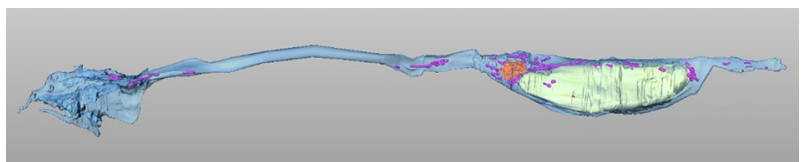

Movie 4. Three-dimensional reconstruction image of a migratory neuroblast during leading process extension. Green, yellow, orange, and magenta represent centrioles, nucleus, Golgi apparatus, and mitochondria, respectively.
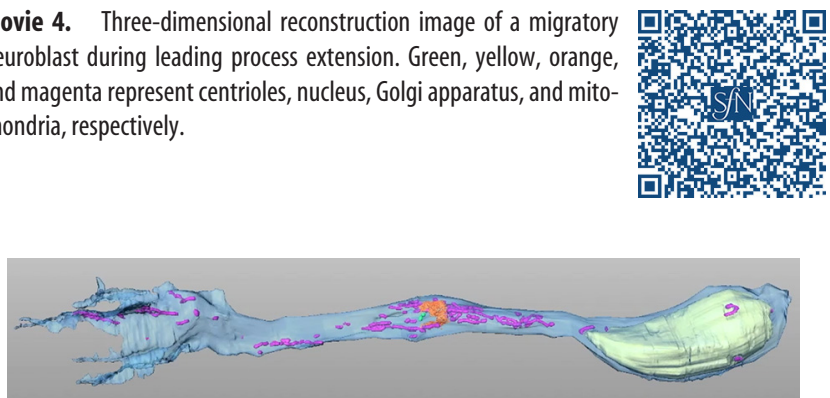

Movie 5. Three-dimensional reconstruction image of a migratory neuroblast in the swelling-formation phase. Green, yellow, orange, and magenta represent centrioles, nucleus, Golgi apparatus, and mitochondria, respectively.
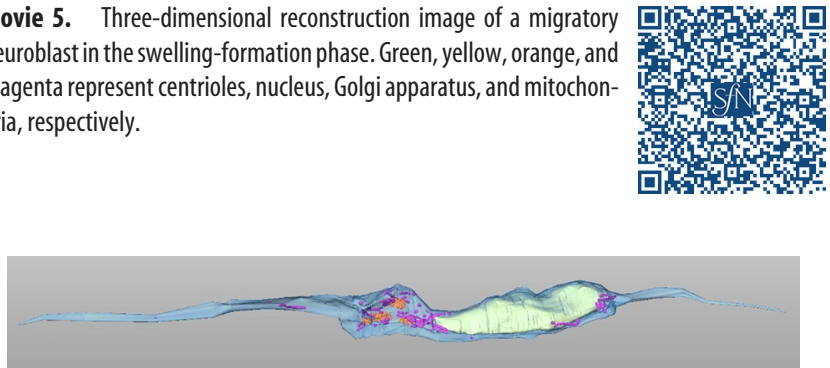

Movie 6. Three-dimensional reconstruction image of a migratory neuroblast during somal translocation. Green, yellow, orange, and magenta represent centrioles, nucleus, Golgi apparatus, and mitochondria, respectively.
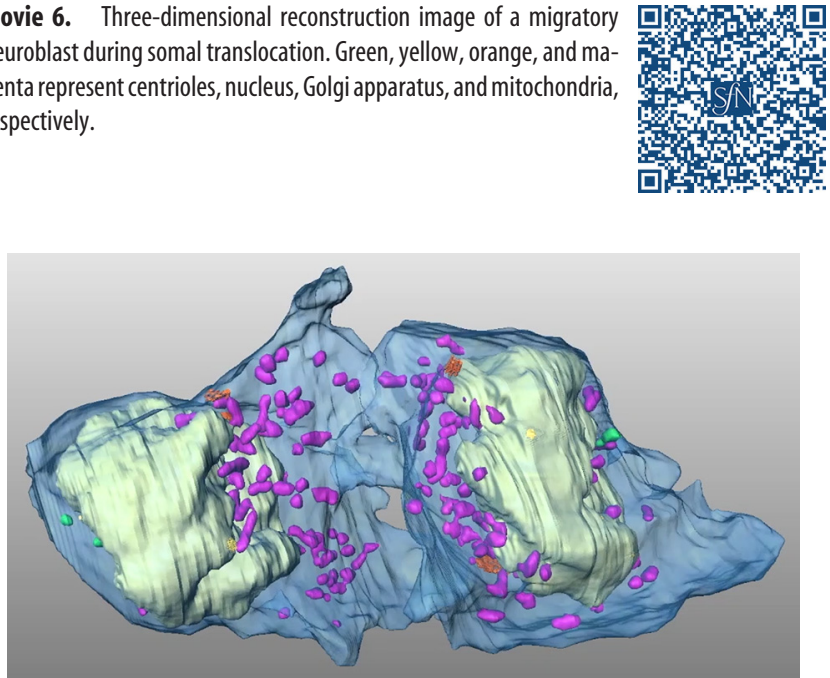

Movie 7. Three-dimensional reconstruction image of dividing neuroblasts. Blue and yellow represent the cell contour and divided nuclei, respectively. Green, orange, and magenta represent centrioles, Golgi apparatus, and mitochondria, respectively.

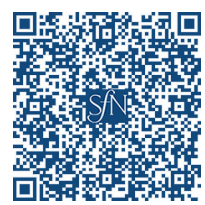

nonextended primary cilium in the $\mathrm{OB}$, and both were longer than their counterparts in the RMS (Fig. 7C). Together, these results suggest that the elongation and extension of primary cilium are promoted in individual decelerating neuroblasts in the OB.

To understand the extracellular microenvironment of the extended primary cilium in migrating neuroblasts in the RMS and $\mathrm{OB}$, we identified neural structures contacting the extended primary cilium in the RMS and OB. In the RMS, each extended primary cilium made contact with a neighboring neuroblast $(28$ 

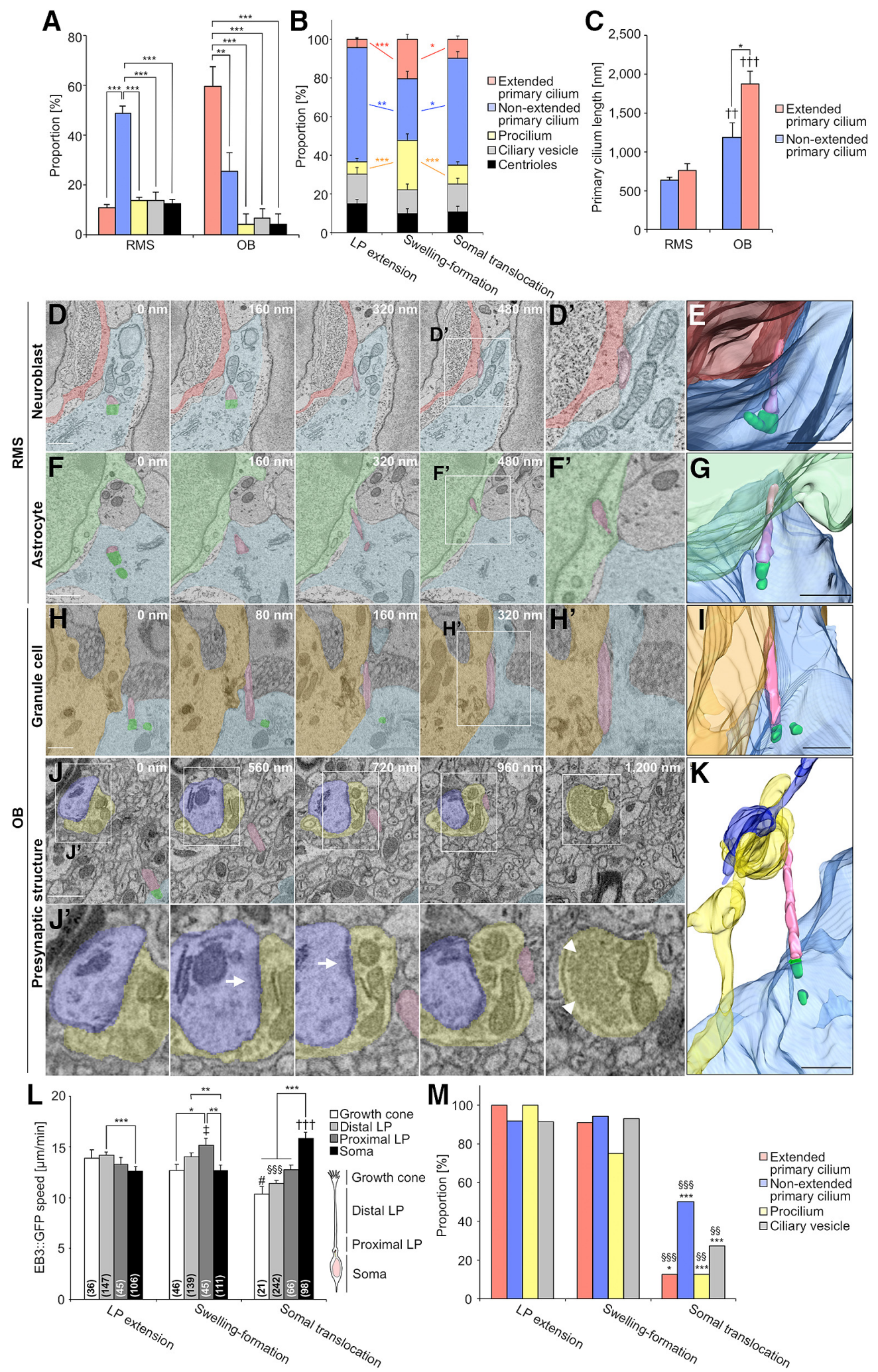

Figure 7. Changes in subcellular localization, length, and orientation of the primary cilium during saltatory movement of migrating neuroblasts in the adult RMS. $A$, The proportions of immature migratory neuroblasts that have centrioles, a ciliary vesicle, a procilium, a nonextended primary cilium, and an extended primary cilium in the adult RMS $\left(F_{(4,20)}=56.59, p=1.3 \times 10^{-10}\right.$, one-way ANOVA; centrioles vs nonextended primary cilium, $p<0.0000001$, ciliary vesicle vs nonextended primary cilium, $p<0.0000001$, procilium vs nonextended primary cilium, $p<0.0000001$, extended primary cilium vs nonextended primary cilium, $p<0.0000001$, Tukey-Kramer test; $n=5$ mice) and $O B\left(F_{(4,10)}=17.28, p=0.00017\right.$, one-way ANOVA; centrioles vs extended primary cilium, $p=0.00034$, ciliary vesicle vs extended primary cilium, $p=0.0005$, procilium vs extended primary cilium, $p=0.00034$, nonextended primary cilium vs extended primary cilium, $p=0.012$, Tukey-Kramer test; $n=3$ mice). $\boldsymbol{B}$, The proportions of neuroblasts that have centrioles, a ciliary vesicle, a procilium, a nonextended primary cilium, and an extended primary cilium during leading-process (LP) extension phase, the swelling-formation phase, and somal translocation phase in the adult RMS $\left(F_{\text {phase }(2,12)}=-0.004, p_{\text {phase }}=1, F_{\text {class }}(4,48)=75.52, p_{\text {class }}<2.2 \times 10^{-16}\right.$,

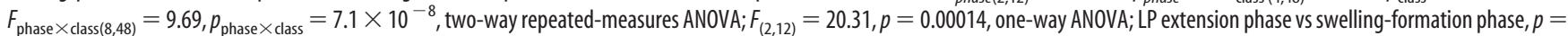
0.00092 , somal translocation phase vs swelling-formation phase, $p=0.0045$, in procilium; $F_{(2,12)}=13.02, p=0.00099$, one-way ANOVA; LP extension (Figure legend continues.) 


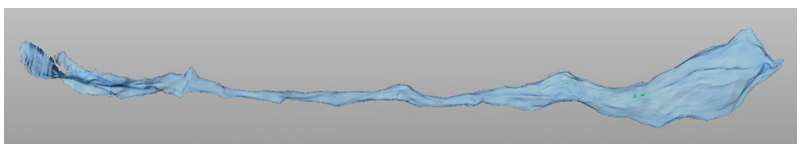

Movie 8. Three-dimensional reconstruction image of a neuroblast having centrioles. Blue and green represent the cell contour and centrioles, respectively.

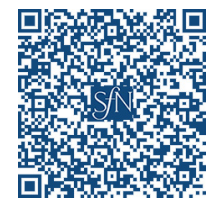

of 36 cells) or astrocyte ( 8 of 36 cells) (Fig. $7 D-G$ ). In the granule cell layer of the $\mathrm{OB}$, the extended primary cilium of neuroblasts directly contacted neurites of mature neurons (14 of 14 cells). Close observation shows that each primary cilium of these neu-

$\leftarrow$

(Figure legend continued.) phase vs swelling-formation phase, $p=0.0071$, somal translocation phase vs swelling-formation phase, $p=0.02$, in nonextended primary cilium; $F_{(2,12)}=$ $18.55, p=0.00021$, one-way ANOVA; LP extension phase vs swelling-formation phase, $p=$ 0.00088 , somal translocation phase vs swelling-formation phase, $p=0.023$, in extended primary cilium; Tukey-Kramer test; $n=5$ mice). ${ }^{*} p<0.05$; ${ }^{* *} p<0.01$; ${ }^{* * *} p<0.005$; adjusted with Bonferroni correction. $C$, Length of the nonextended and extended primary cilium in immature neuroblasts in the adult RMS and $O B$ ( $R M S$ v $O B, W=869.5 p=0.012$, in nonextended primary cilium; Mann-Whitney $U$ test; $t_{(48)}=6.68, p=2.3 \times 10^{-8}$, in extended primary cilium; nonextended primary cilium vs extended primary cilium, $t_{(19)}=-2.7, p=0.014$, in $O B$; unpaired $t$ test; $n=3$ mice each). $\boldsymbol{D}-\boldsymbol{K}$, Sequential SBF-SEM images and $3 \mathrm{D}$ constructions of extended primary cilium contacting a neighboring neuroblast $(\boldsymbol{D}, \boldsymbol{E}$, red), astrocyte $(\boldsymbol{F}, \boldsymbol{G}$, green), granule cell $\left(\boldsymbol{H}, \boldsymbol{I}\right.$, orange), and presynaptic structure $\left(\boldsymbol{J}, \boldsymbol{K}\right.$, yellow). $\boldsymbol{J}^{\prime}$, White arrows and arrowheads indicate postsynaptic density and synaptic vesicles, respectively. $L$, EB3::GFP speed in the growth cone, distal/proximal leading process, and soma during saltatory movement of cultured chain-forming migrating neuroblasts $\left(\chi_{(3)}^{2}=14.67, p=0.0021\right.$, Kruskal-Wallis test; distal leading process vs soma, $t(\infty)=3.66, p=0.0014$, in LP extension phase; $\chi_{(3)}^{2}=17.77, p=$ 0.00049 , Kruskal-Wallis test; distal leading process vs soma, $t(\infty)=3.13, p=0.0094$, growth cone vs proximal leading process, $t(\infty)=2.84, p=0.023$, proximal leading process vs soma, $t(\infty)=3.12, p=0.0097$, in swelling-formation phase; $\chi_{(3)}^{2}=53.72, p=1.3 \times 10^{-11}$, Kruskal-Wallis test; distal leading process vs soma, $t(\infty)=6.87, p=4.0 \times 10^{-11}$, growth cone vs soma, $t(\infty)=4.19, p=1.6 \times 10^{-4}$, proximal leading process vs soma, $t(\infty)=3.45$ $p=3.1 \times 10^{-3}$, in somal translocation phase; $\chi_{(2)}^{2}=8.61, p=0.0014$, Kruskal-Wallis test; LP extension phase vs somal translocation phase, $t(\infty)=2.71, p=0.0018$, swelling-formation phase vs somal translocation phase, $t(\infty)=2.4, p=0.043$, in growth cone; $\chi_{(2)}^{2}=7.21, p<$ $2.2 \times 10^{-16}$, Kruskal-Wallis test; LP extension phase vs somal translocation phase, $t(\infty)=$ $7.46, p=2.7 \times 10^{-13}$, swelling-formation phase vs somal translocation phase, $t(\infty)=6.55$, $p=1.8 \times 10^{-10}$, in distal leading process; $\chi_{(2)}^{2}=8.8, p=0.0012$, Kruskal-Wallis test; $L P$ extension phase vs swelling-formation phase, $t(\infty)=2.43, p=0.04$, swelling-formation phase vs somal translocation phase, $t(\infty)=2.69, p=0.019$, in proximal leading process; $\chi_{(3)}^{2}$ $=22.18, p=1.5 \times 10^{-5}$, Kruskal-Wallis test; LP extension phase vs somal translocation phase, $t(\infty)=4.1, p=0.00012$, swelling-formation phase vs somal translocation phase, $t(\infty)=4.11, p=0.00012$, in soma; Steel-Dwass test). ${ }^{*} p<0.05 ;{ }^{* *} p<0.01 ;{ }^{* * *} p<0.005$; ${ }^{\#} p<0.05$; versus $L P$ extension phase and swelling-formation phase in growth cone. $\S \S \S p<$ 0.005 , versus $L P$ extension phase and swelling-formation phase in distal leading process. $\neq p<$ 0.05 , versus $L P$ extension phase and somal translocation phase in proximal leading process. $t+t p<0.005$, versus LP extension phase and swelling-formation phase in soma. Values in parentheses indicate the number of events from eight cells in three independent cultures. $\boldsymbol{M}$, The proportions of immature and mature primary cilium that orient posteriorly under LP extension phase, the swelling-formation phase, and somal translocation phase in the adult RMS ( $p=0.00015$, Fisher's exact test, somal translocation phase vs swelling-formation phase, $p=$ 0.0064 , LP extension phase vs somal translocation phase, $p=0.00098$, in ciliary vesicle; $p=$ 0.00015 , Fisher's exact test; somal translocation phase vs swelling-formation phase, $p=0.0078$, LP extension phase vs somal translocation phase, $p=0.00075$, in procilium; $p=$ $6.2 \times 10^{-8}$, Fisher's exact test, somal translocation phase vs swelling-formation phase, $p=$ 0.00012 , LP extension phase vs somal translocation phase, $p=9.4 \times 10^{-7}$, in nonextended primary cilium; $p=9.5 \times 10^{-5}$, Fisher's exact test, somal translocation phase vs swellingformation phase, $p=0.00039$, LP extension phase vs somal translocation phase, $p=0.014$, in extended primary cilium; $n=5$ mice). ${ }^{*} p<0.05 ;{ }^{* * *} p<0.005$; versus LP extension phase. $\S p<0.05 ; \S \S \S p<0.005$; versus swelling-formation phase. Error bars indicate mean \pm SEM. Scale bars, $1 \mu \mathrm{m}$.

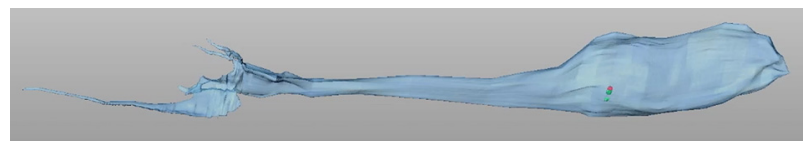

Movie 9. Three-dimensional reconstruction image of a neuroblast having a ciliary vesicle. Blue, green, and magenta represent the cell contour, centrioles, and ciliary vesicle, respectively.
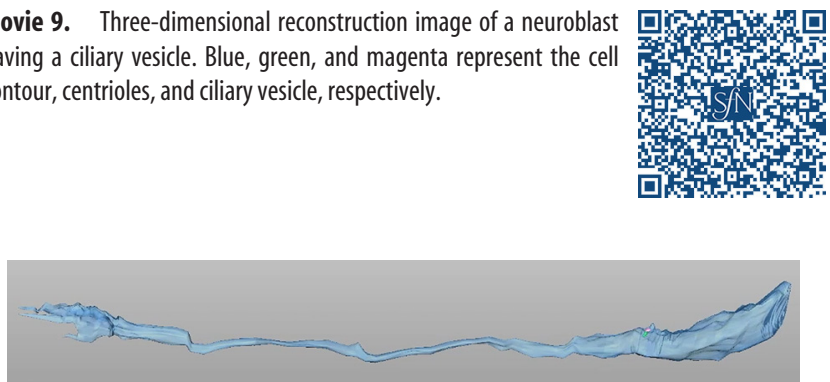

Movie 10. Three-dimensional reconstruction image of a neuroblast having a procilium. Blue, green, and magenta represent the cell contour, centrioles, and ciliary membrane, respectively.
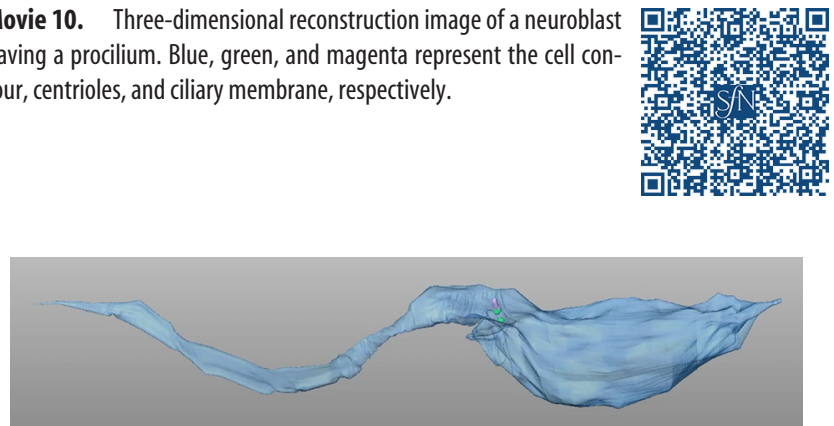

Movie 11. Three-dimensional reconstruction image of a neuroblast having a nonextended primary cilium. Blue, green, and magenta represent the cell contour, centrioles, and ciliary membrane, respectively.
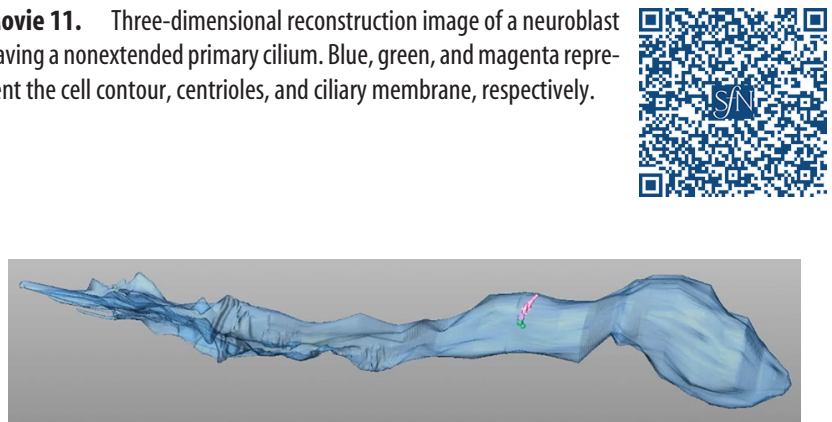

Movie 12. Three-dimensional reconstruction image of a neuroblast having an extended primary cilium. Blue, green, and magenta represent cell contour, centrioles, and ciliary membrane, respectively.

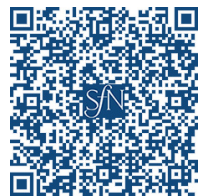

roblasts was making contact with the soma of granule cells (3 of 14 cells) or with presynaptic structures containing synaptic vesicles that had formed on the neurites ( 11 of 14 cells) (Fig. $7 \mathrm{H}-\mathrm{K}$ ). These results suggest that the extended primary cilium in a neuroblast interacts with its surrounding cells during migration in the RMS and OB.

Finally, we analyzed the dynamics of MT polymerization in each cellular domain during saltatory movement of cultured chain-forming neuroblasts, and compared the data with the orientation of the primary cilium analyzed using SBF-SEM. The highest polymerization levels of MTs labeled with EB3::GFP (Sawada et al., 2018) were observed in the distal leading process during leading-process extension phase, in the proximal leading process during the swelling-formation phase, and in the soma during somal translocation phase (Fig. $7 L$; Movie 13). On the other hand, quantification of ciliary orientation based on the SBF-SEM images (Fig. $6 D^{\prime}, F^{\prime}, H^{\prime}, J^{\prime}$ ) revealed that the majority 


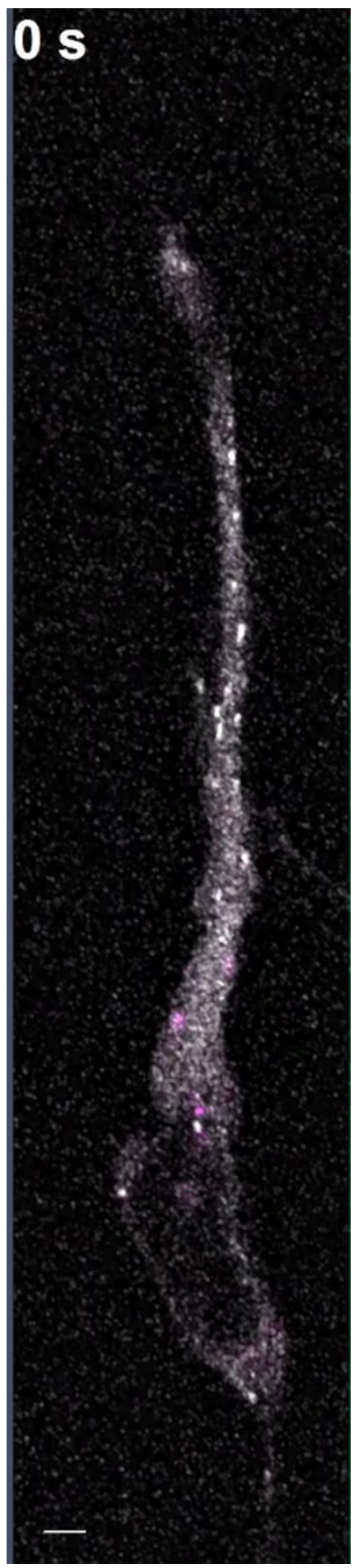

Movie 13. Time-lapse imaging of EB3::GFP and dTomato::Cent2 during saltatory movement of a cultured V-SVZ-derived migrating neuroblast. The migratory behavior of an EB3::GFP- (white) and dTomato:: Cent2 (magenta)-expressing V-SVZ-derived neuroblast was recorded at 4 s intervals by superresolution microscopy. Leading-process extension phase, $0-440 \mathrm{~s}$; swelling-formation phase, $444-1280 \mathrm{~s}$; somal translocation phase, $1284-1920$ s. Scale bar, $2 \mu \mathrm{m}$.

of migrating neuroblasts ( $80 \%-100 \%)$ of the leading-process extension and swelling-formation phases had a posteriorly oriented primary cilium regardless of the ciliary type, whereas migrating neuroblasts of the somal translocation phase had an anteriorly oriented primary cilium (Fig. $7 M$ ). The orientation of the primary cilium in the direction opposite to the cellular domain with the highest levels of MT polymerization suggests that ciliary orientation is related to the difference in the local MT polymerization levels in each migration phase rather than to ciliary maturation states.

\section{Discussion}

SBF-SEM analyses of migrating neuroblasts and the primary cilium in vivo

Time-lapse imaging of V-SVZ-derived migrating neuroblasts reveals each step of their saltatory movement (Schaar and McConnell, 2005; Shinohara et al., 2012; Ota et al., 2014; Sawada et al., 2018). Here, by analyzing the presence/absence of swelling and growth cone morphology, we could identify the migration phases of immature neuroblasts both in time-lapse imaging and in SBFSEM analyses. Moreover, 3D reconstruction of mitochondria showed changes in their distribution in migration phases, which is consistent with a previous study in MGE-derived cortical interneurons (Lin-Hendel et al., 2016). Since dynamic changes in cellular morphology in migrating neuroblasts are supported by mitochondrial ATP production (Lin-Hendel et al., 2016), it is possible that mitochondria accumulate in subcellular regions with high-energy demands, such as the swelling.

SBF-SEM or another type of 3D-SEM, focused ion beamSEM, has previously been used to analyze the fine morphology of the primary cilium (Paridaen et al., 2013; Insinna et al., 2019) and the molecular mechanisms of ciliogenesis (David et al., 2014; Buskin et al., 2018). Although our SBF-SEM analyses could not clearly visualize MT structures, we successfully identified primary cilium structures by detecting their basal bodies and associated membrane structures, whose electron density was strengthened by the reduced osmium-thiocarbohydrazideosmium method (Seligman et al., 1966; Willingham and Rutherford, 1984). Our ultrastructural SBF-SEM images for different stages of ciliogenesis should provide a basis for analysis of the primary cilium in other cells in the brain or in other tissues under physiological and pathological conditions.

\section{Unique morphology and dynamics of the primary cilium in V-SVZ-derived migrating neuroblasts}

In contrast to other immature neurons migrating in the developing brain, the V-SVZ-derived neuroblasts are unique in that they are still mitotic and migrating in a chain in the RMS. Our observations suggest that ciliogenesis in migrating neuroblasts is transiently suppressed during mitosis (Fig. 8A), as reported in other mitotic cells (Malicki and Johnson, 2017). In addition, a small portion of proliferating $\mathrm{Dcx}^{+}$cells has an Arl $13 \mathrm{~b}^{+}$primary cilium, even in the $M$ phase. It is possible that the status of the primary cilium in migrating neuroblasts is influenced by dynamic changes in their cellular circumstances, including those in the cell cycle and saltatory movement.

Our live imaging and EM analyses revealed that migrating neuroblasts in the postnatal RMS possess a primary cilium in the cytosol, which is transiently extended from the cell surface before somal translocation (Fig. 8B). Similar subcellular translocation of the primary cilium during migration has been reported in MGEderived interneurons (Baudoin et al., 2012), suggesting that ciliogenesis in interneurons is regulated by common mechanisms in the embryonic and postnatal brain. Interestingly, the "nonextended" primary cilium in immature neuroblasts appeared to have access to the extracellular environment, which has been 
A Proliferation of chain-forming neuroblasts in the RMS

Non-proliferating neuroblasts

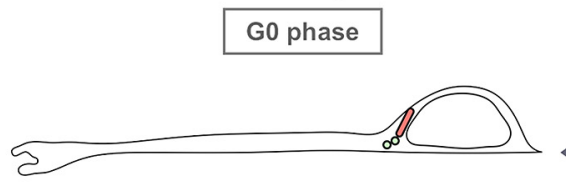

Suppression of ciliogenesis
Proliferating neuroblasts

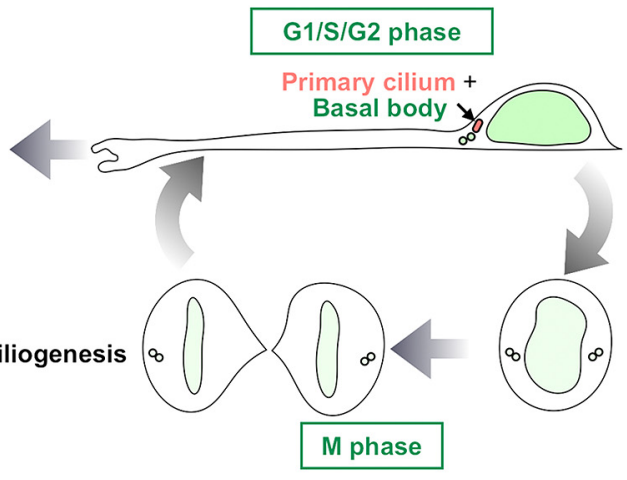

B Chain-forming neuroblasts in the RMS (Maintenance of migration)

\section{FLP-bearing neuroblasts in the $O B$ (Deceleration of migration)}
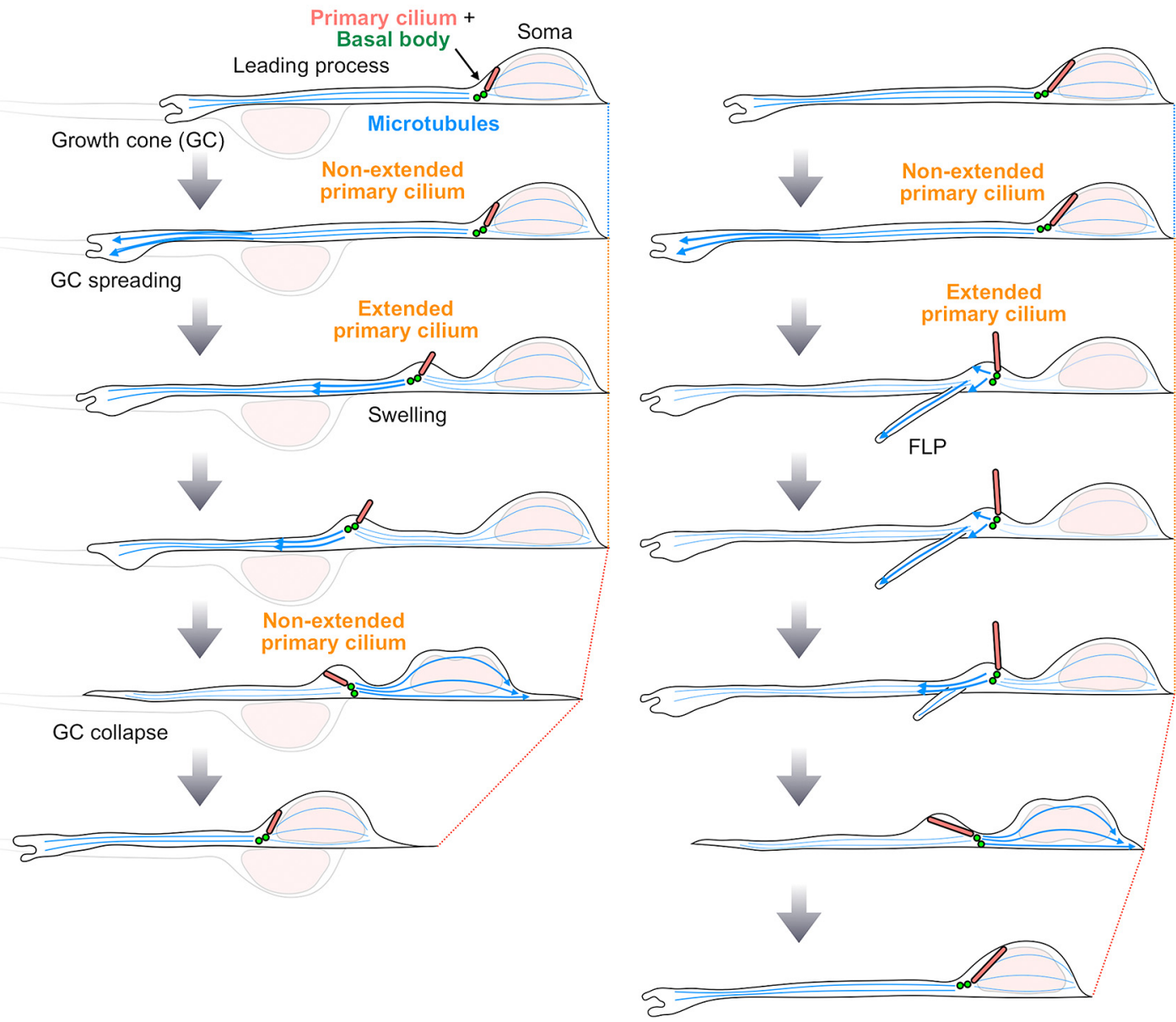

Figure 8. Spatiotemporal regulation of the primary cilium in V-SVZ-derived migrating neuroblasts in the postnatal brain. $A$, The primary cilium in proliferating neuroblasts in the postnatal RMS. Ciliogenesis is suppressed during mitosis (green nuclei) of V-SVZ-derived migrating neuroblasts in the RMS. B, The primary cilium in migration of chain-forming neuroblasts in the RMS. The primary cilium (pink) is submerged in the cytosol and orients posteriorly during leading process extension, when a spread growth cone is observed. During the swelling-formation phase, the primary cilium is extended from the cell surface and moves forward within the swelling. The primary cilium is not extended when neuroblasts translocate their soma. The orientation of the primary cilium is related to active MT polymerization (bold blue arrows) in migration phases. C, The primary cilium in migration of FLP-bearing neuroblasts in the postnatal OB. The FLP is transiently formed from the proximal part of the leading process before somal translocation (Sawada et al., 2018). FLP formation coincides with active and local MT reorganization in the proximal leading process (bold blue arrows), which maintains migration termination in neuroblasts. The elongation and extension of the primary cilium are promoted in individual decelerating neuroblasts in the $\mathrm{OB}$.

reported in other types of mature cells (Sorokin, 1962; Dahl, 1967; Smith et al., 1969; Mazo et al., 2016).

In MGE-derived migrating interneurons, extracellular extension of the primary cilium could be regulated by fusion and fission of Golgi-derived ciliary vesicles (Baudoin et al., 2012). A recent study suggests that the submerged state of the primary cilium in the cytosol is maintained by association of daughter centriole with the Golgi apparatus in retinal pigmental epithelial cells (Mazo et al., 2016). It remains unknown whether these mechanisms are involved in maintenance of the nonex- 
tended state of the primary cilium in postnatal-born migrating neuroblasts.

The FLP-bearing individual neuroblasts decelerating in the $\mathrm{OB}$ restrict forward movement of the primary cilium in the leading process and promote cilium extension from the cell surface (Fig. 8C), which is distinct from chain-forming neuroblasts in the RMS. These changes in primary cilium dynamics may be related to termination of migration and initiation of differentiation in the $\mathrm{OB}$, which are suppressed by homophilic chain formation and/or efficient migration in the RMS.

\section{Possible functions of the primary cilium in V-SVZ-derived migrating neuroblasts}

Our SBF-SEM results showed that $88 \%$ of immature neuroblasts in the adult RMS possess ciliary structures on their mother centrioles. Moreover, MTs were observed to be extended radially from the basal body of the primary cilium in migrating neuroblasts. Similar relationships between MTs and the basal body of the primary cilium have also been reported in fibroblasts (Albrecht-Buehler and Bushnell, 1980; Schrøder et al., 2011) and enteric neurons (Luesma et al., 2013). These findings suggest that the basal body of the primary cilium acts as an MT-organizing center in the V-SVZ-derived migrating neuroblasts, which is consistent with previous studies (Tsai et al., 2007; Baudoin et al., 2012).

We found that the primary cilium orients posteriorly when neuroblasts actively polymerize MTs in the leading process, whereas it orients anteriorly when neuroblasts actively form a perinuclear MT cage (Fig. $8 B$ ). Thus, it is possible that active MT polymerization generates traction force that pulls the basal body, which would affect the ciliary orientation during saltatory movement. During neuroblast deceleration, the FLP induces local MT reorganization in the proximal leading process, which can decrease the MT polymerization-based traction force and sustain extension of the primary cilium in FLP-bearing neuroblasts. Together, these results suggest that the dynamics of the basal body and primary cilium are regulated by MT dynamics in saltatory movement of migrating neuroblasts.

Kif3A promotes ciliogenesis by regulating axoneme elongation and subdistal appendage formation in the basal body (Kodani et al., 2013). However, Kif3A also has nonciliary functions, such as transport of $\mathrm{N}$-cadherin (Teng et al., 2005; Ichinose et al., 2015) and modulation of Wnt signaling (Corbit et al., 2008). Therefore, defective neuroblast migration caused by dnKif3 A expression does not directly indicate the function of the primary cilium in neuroblast migration in the RMS. The KD of IFT88, which contributes to ciliogenesis independently of Kif3A (Pazour et al., 2000), also disrupted migration of V-SVZ-derived neuroblasts, similar to MGE-derived cortical interneurons (Baudoin et al., 2012). However, since the V-SVZ-derived migrating neuroblasts are mitotic, nonciliary functions of IFT88 during mitosis, including the regulation of spindle orientation (Robert et al., 2007; Delaval et al., 2011; Borovina and Ciruna, 2013), may affect neuronal distribution. Thus, our results suggest that disrupted neuroblast migration resulting from inhibition of Kif3 $\mathrm{A}$ and IFT88 is, at least in part, attributable to the defects of primary cilium function in the V-SVZ-derived migrating neuroblasts.

The primary cilium contains signaling receptors, such as Smoothened and 5HT6, on its membrane, which act as chemical sensors to regulate migration and maturation of cortical interneurons (Riccio et al., 2009; Baudoin et al., 2012; Higginbotham et al., 2012; Guo et al., 2017). Shh signaling promotes neuroblast migration in the V-SVZ (Angot et al., 2008). More- over, a recent study suggests that serotonin controls speed and directionality of the V-SVZ-derived migrating neuroblasts (García-González et al., 2017). Whereas 5HT6::GFP localized only in the primary cilium, 5HT3A::GFP was detectable not only in the cilium but also in the cell body (Fig. $1 J, L$ ). Furthermore, 5HT6 and 5HT3A are a GPCR and a ligand-gated ion channel, respectively (Derkach et al., 1989; Monsma et al., 1993; Ruat et al., 1993). Therefore, it is possible that these two types of serotonin receptors coordinately regulate neuroblast migration in the adult RMS and OB via distinct signaling pathways. Serotonin fibers extending along the RMS-OB pathway (García-González et al., 2017) and the presence of a primary cilium in migrating neuroblasts are widely conserved among vertebrates. Since the postnatal-born neuroblasts must migrate over a long distance within the mature neuronal circuitry, which is distinct from embryonic neurons, they may need to continuously sense extracellular cues through the primary cilium for their efficient migration.

Migration and maturation of neuroblasts in the OB are affected by both olfactory (Petreanu and Alvarez-Buylla, 2002; Saghatelyan et al., 2004; Yamaguchi and Mori, 2005) and centrifugal (Kaneko et al., 2006; García-González et al., 2017) inputs. Interestingly, the extended primary cilium in individually migrating neuroblasts made direct contact with presynaptic structures in the $\mathrm{OB}$ (Fig. $7 \mathrm{~J}, \mathrm{~K}$ ). If the extended primary cilium has the potential to transduce electrical activity, as in the renal collecting duct epithelium (Liu et al., 2018), it may contribute to activity-dependent neuroblast migration and maturation in the OB.

Although the primary cilium has also been proposed to act as a mechanosensor (Malicki and Johnson, 2017), it remains unknown whether the primary cilium in migrating neuroblasts mediates mechanosensing. In retinal pigment epithelial cells, whereas the submerged primary cilium was not affected by fluid flow, the surfaced primary cilium, induced by C-Nap1/CEP128 deficiencies, bent in response to fluid flow (Mazo et al., 2016). If the primary cilium in migrating neuroblasts is able to transduce mechanostimulation, its activity could be altered between nonextended and extended states, and affected by cell-cell contact that changes dynamically during chain migration.

In conclusion, this study suggests that primary cilium dynamics is related to the proliferation and saltatory movement of V-SVZ-derived migrating neuroblasts. Our detailed description of the ultrastructural features and dynamics of primary cilium in migrating neuroblasts will be useful for future studies, which will clarify the functional significance of the primary cilium and novel regulatory mechanisms for neuroblast migration.

\section{References}

Albrecht-Buehler G, Bushnell A (1980) The ultrastructure of primary cilia in quiescent 3T3 cells. Exp Cell Res 126:427-437.

Amador-Arjona A, Elliott J, Miller A, Ginbey A, Pazour GJ, Enikolopov G, Roberts AJ, Terskikh AV (2011) Primary cilia regulate proliferation of amplifying progenitors in adult hippocampus: implications for learning and memory. J Neurosci 31:9933-9944.

Angot E, Loulier K, Nguyen-Ba-Charvet KT, Gadeau AP, Ruat M, Traiffort E (2008) Chemoattractive activity of sonic hedgehog in the adult subventricular zone modulates the number of neural precursors reaching the olfactory bulb. Stem Cells 26:2311-2320.

Arellano JI, Guadiana SM, Breunig JJ, Rakic P, Sarkisian MR (2012) Development and distribution of neuronal cilia in mouse neocortex. J Comp Neurol 520:848-873.

Arvidsson A, Collin T, Kirik D, Kokaia Z, Lindvall O (2002) Neuronal replacement from endogenous precursors in the adult brain after stroke. Nat Med 8:963-970. 
Baudoin JP, Viou L, Launay PS, Luccardini C, Espeso Gil S, Kiyasova V, Irinopoulou T, Alvarez C, Rio JP, Boudier T, Lechaire JP, Kessaris N, Spassky N, Métin C (2012) Tangentially migrating neurons assemble a primary cilium that promotes their reorientation to the cortical plate. Neuron 76:1108-1122.

Belevich I, Joensuu M, Kumar D, Vihinen H, Jokitalo E (2016) Microscopy image browser: a platform for segmentation and analysis of multidimensional datasets. PLoS Biol 14:e1002340.

Bellion A, Baudoin JP, Alvarez C, Bornens M, Métin C (2005) Nucleokinesis in tangentially migrating neurons comprises two alternating phases: forward migration of the Golgi/centrosome associated with centrosome splitting and myosin contraction at the rear. J Neurosci 25:5691-5699.

Belvindrah R, Nissant A, Lledo PM (2011) Abnormal neuronal migration changes the fate of developing neurons in the postnatal olfactory bulb. J Neurosci 31:7551-7562.

Bishop GA, Berbari NF, Lewis J, Mykytyn K (2007) Type III adenylyl cyclase localizes to primary cilia throughout the adult mouse brain. J Comp Neurol 505:562-571.

Borovina A, Ciruna B (2013) IFT88 plays a cilia- and PCP-independent role in controlling oriented cell divisions during vertebrate embryonic development. Cell Rep 5:37-43.

Brailov I, Bancila M, Brisorgueil MJ, Miquel MC, Hamon M, Vergé D (2000) Localization of 5-HT(6) receptors at the plasma membrane of neuronal cilia in the rat brain. Brain Res 872:271-275.

Breunig JJ, Sarkisian MR, Arellano JI, Morozov YM, Ayoub AE, Sojitra S, Wang B, Flavell RA, Rakic P, Town T (2008) Primary cilia regulate hippocampal neurogenesis by mediating sonic hedgehog signaling. Proc Natl Acad Sci U S A 105:13127-13132.

Briggman KL, Bock DD (2012) Volume electron microscopy for neuronal circuit reconstruction. Curr Opin Neurobiol 22:154-161.

Buskin A, Zhu L, Chichagova V, Basu B, Mozaffari-Jovin S, Dolan D, Droop A, Collin J, Bronstein R, Mehrotra S, Farkas M, Hilgen G, White K, Pan KT, Treumann A, Hallam D, Bialas K, Chung G, Mellough C, Ding Y, et al. (2018) Disrupted alternative splicing for genes implicated in splicing and ciliogenesis causes PRPF31 retinitis pigmentosa. Nat Commun 9:4234.

Capilla-Gonzalez V, Cebrian-Silla A, Guerrero-Cazares H, Garcia-Verdugo JM, Quiñones-Hinojosa A (2014) Age-related changes in astrocytic and ependymal cells of the subventricular zone. Glia 62:790-803.

Caspary T, Larkins CE, Anderson KV (2007) The graded response to sonic hedgehog depends on cilia architecture. Dev Cell 12:767-778.

Chen JL, Chang CH, Tsai JW (2019) Gli2 rescues delays in brain development induced by Kif3a dysfunction. Cereb Cortex 29:751-764.

Corbit KC, Shyer AE, Dowdle WE, Gaulden J, Singla V, Chen MH, Chuang PT, Reiter JF (2008) Kif3a constrains beta-catenin-dependent wnt signalling through dual ciliary and non-ciliary mechanisms. Nat Cell Biol 10:70-76.

Dahl HA (1967) On the cilium cell relationship in the adenohypophysis of the mouse. Z Zellforsch Mikrosk Anat 83:169-177.

Danilov AI, Gomes-Leal W, Ahlenius H, Kokaia Z, Carlemalm E, Lindvall O (2009) Ultrastructural and antigenic properties of neural stem cells and their progeny in adult rat subventricular zone. Glia 57:136-152.

David A, Liu F, Tibelius A, Vulprecht J, Wald D, Rothermel U, Ohana R, Seitel A, Metzger J, Ashery-Padan R, Meinzer HP, Gröne HJ, Izraeli S, Krämer A (2014) Lack of centrioles and primary cilia in $\operatorname{STIL}(-/-)$ mouse embryos. Cell Cycle 13:2859-2868.

Delaval B, Bright A, Lawson ND, Doxsey S (2011) The cilia protein IFT88 is required for spindle orientation in mitosis. Nat Cell Biol 13:461-468.

Denk W, Briggman KL, Helmstaedter M (2012) Structural neurobiology: missing link to a mechanistic understanding of neural computation. Nat Rev Neurosci 13:351-358.

Derkach V, Surprenant A, North RA (1989) 5-HT3 receptors are membrane ion channels. Nature 339:706-709.

Doetsch F, García-Verdugo JM, Alvarez-Buylla A (1997) Cellular composition and three-dimensional organization of the subventricular germinal zone in the adult mammalian brain. J Neurosci 17:5046-5061.

Ford MJ, Yeyati PL, Mali GR, Keighren MA, Waddell SH, Mjoseng HK, Douglas AT, Hall EA, Sakaue-Sawano A, Miyawaki A, Meehan RR, Boulter L, Jackson IJ, Mill P, Mort RL (2018) A cell/cilia cycle biosensor for single-cell kinetics reveals persistence of cilia after $\mathrm{G}_{1} / \mathrm{S}$ transition is a general property in cells and mice. Dev Cell 47:509-523.e5.

Fujikake K, Sawada M, Hikita T, Seto Y, Kaneko N, Herranz-Perez V, Dohi N,
Homma N, Osaga S, Yanagawa Y, Akaike T, García-Verdugo JM, Hattori M, Sobue K, Sawamoto K (2018) Detachment of chain-forming neuroblasts by fyn-mediated control of cell-cell adhesion in the postnatal brain. J Neurosci 38:4598-4609.

García-González D, Khodosevich K, Watanabe Y, Rollenhagen A, Lübke JH, Monyer H (2017) Serotonergic projections govern postnatal neuroblast migration. Neuron 94:534-549.e9.

Guadiana SM, Semple-Rowland S, Daroszewski D, Madorsky I, Breunig JJ, Mykytyn K, Sarkisian MR (2013) Arborization of dendrites by developing neocortical neurons is dependent on primary cilia and type 3 adenylyl cyclase. J Neurosci 33:2626-2638.

Guemez-Gamboa A, Coufal NG, Gleeson JG (2014) Primary cilia in the developing and mature brain. Neuron 82:511-521.

Guo J, Otis JM, Higginbotham H, Monckton C, Cheng J, Asokan A, Mykytyn K, Caspary T, Stuber GD, Anton ES (2017) Primary cilia signaling shapes the development of interneuronal connectivity. Dev Cell 42:286-300.e4.

Han YG, Spassky N, Romaguera-Ros M, Garcia-Verdugo JM, Aguilar A, Schneider-Maunoury S, Alvarez-Buylla A (2008) Hedgehog signaling and primary cilia are required for the formation of adult neural stem cells. Nat Neurosci 11:277-284.

Higginbotham H, Bielas S, Tanaka T, Gleeson JG (2004) Transgenic mouse line with green-fluorescent protein-labeled centrin 2 allows visualization of the centrosome in living cells. Transgenic Res 13:155-164.

Higginbotham H, Eom TY, Mariani LE, Bachleda A, Hirt J, Gukassyan V, Cusack CL, Lai C, Caspary T, Anton ES (2012) Arl13b in primary cilia regulates the migration and placement of interneurons in the developing cerebral cortex. Dev Cell 23:925-938.

Hikita T, Ohno A, Sawada M, Ota H, Sawamoto K (2014) Rac1-mediated indentation of resting neurons promotes the chain migration of new neurons in the rostral migratory stream of post-natal mouse brain. J Neurochem 128:790-797.

Ichinose S, Ogawa T, Hirokawa N (2015) Mechanism of activity-dependent cargo loading via the phosphorylation of KIF3A by PKA and CaMKIIa. Neuron 87:1022-1035.

Ikeda M, Hirota Y, Sakaguchi M, Yamada O, Kida YS, Ogura T, Otsuka T, Okano H, Sawamoto K (2010) Expression and proliferation-promoting role of diversin in the neuronally committed precursor cells migrating in the adult mouse brain. Stem Cells 28:2017-2026.

Insinna C, Lu Q, Teixeira I, Harned A, Semler EM, Stauffer J, Magidson V, Tiwari A, Kenworthy AK, Narayan K, Westlake CJ (2019) Investigation of F-BAR domain PACSIN proteins uncovers membrane tubulation function in cilia assembly and transport. Nat Commun 10:428.

Inta D, Alfonso J, von Engelhardt J, Kreuzberg MM, Meyer AH, van Hooft JA, Monyer H (2008) Neurogenesis and widespread forebrain migration of distinct GABAergic neurons from the postnatal subventricular zone. Proc Natl Acad Sci U S A 105:20994-20999.

Jinnou H, Sawada M, Kawase K, Kaneko N, Herranz-Pérez V, Miyamoto T, Kawaue T, Miyata T, Tabata Y, Akaike T, García-Verdugo JM, Ajioka I, Saitoh S, Sawamoto K (2018) Radial glial fibers support neuronal migration and regeneration after neonatal brain injury. Cell Stem Cell 22:128 137.e9.

Kanda Y (2013) Investigation of the freely available easy-to-use software 'EZR' for medical statistics. Bone Marrow Transplant 48:452-458.

Kaneko N, Okano H, Sawamoto K (2006) Role of the cholinergic system in regulating survival of newborn neurons in the adult mouse dentate gyrus and olfactory bulb. Genes Cells 11:1145-1159.

Kaneko N, Herranz-Pérez V, Otsuka T, Sano H, Ohno N, Omata T, Nguyen HB, Thai TQ, Nambu A, Kawaguchi Y, García-Verdugo JM, Sawamoto K (2018) New neurons use Slit-Robo signaling to migrate through the glial meshwork and approach a lesion for functional regeneration. Sci Adv 4:eaav0618.

Khodosevich K, Seeburg PH, Monyer H (2009) Major signaling pathways in migrating neuroblasts. Front Mol Neurosci 2:7.

Khodosevich K, Zuccotti A, Kreuzberg MM, Le Magueresse C, Frank M, Willecke K, Monyer H (2012) Connexin 45 modulates the proliferation of transit-amplifying precursor cells in the mouse subventricular zone. Proc Natl Acad Sci U S A 109:20107-20112.

Khodosevich K, Lazarini F, von Engelhardt J, Kaneko H, Lledo PM, Monyer H (2013) Connective tissue growth factor regulates interneuron survival and information processing in the olfactory bulb. Neuron 79:1136-1151. Kishimoto N, Alfaro-Cervello C, Shimizu K, Asakawa K, Urasaki A, Nonaka 
S, Kawakami K, Garcia-Verdugo JM, Sawamoto K (2011) Migration of neuronal precursors from the telencephalic ventricular zone into the olfactory bulb in adult zebrafish. J Comp Neurol 519:3549-3565.

Kishimoto N, Asakawa K, Madelaine R, Blader P, Kawakami K, Sawamoto K (2013) Interhemispheric asymmetry of olfactory input-dependent neuronal specification in the adult brain. Nat Neurosci 16:884-888.

Kodani A, Salomé Sirerol-Piquer M, Seol A, Garcia-Verdugo JM, Reiter JF (2013) Kif3a interacts with dynactin subunit p150 glued to organize centriole subdistal appendages. EMBO J 32:597-607.

Kreuzberg M, Kanov E, Timofeev O, Schwaninger M, Monyer H, Khodosevich K (2010) Increased subventricular zone-derived cortical neurogenesis after ischemic lesion. Exp Neurol 226:90-99.

Kumamoto N, Gu Y, Wang J, Janoschka S, Takemaru K, Levine J, Ge S (2012) A role for primary cilia in glutamatergic synaptic integration of adultborn neurons. Nat Neurosci 15:399-405, S1.

Lin-Hendel EG, McManus MJ, Wallace DC, Anderson SA, Golden JA (2016) Differential mitochondrial requirements for radially and non-radially migrating cortical neurons: implications for mitochondrial disorders. Cell Rep 15:229-237.

Liu X, Vien T, Duan J, Sheu SH, DeCaen PG, Clapham DE (2018) Polycystin-2 is an essential ion channel subunit in the primary cilium of the renal collecting duct epithelium. Elife 7:e33183.

Lois C, Alvarez-Buylla A (1994) Long-distance neuronal migration in the adult mammalian brain. Science 264:1145-1148.

Lois C, García-Verdugo JM, Alvarez-Buylla A (1996) Chain migration of neuronal precursors. Science 271:978-981.

Luesma MJ, Cantarero I, Castiella T, Soriano M, Garcia-Verdugo JM, Junquera C (2013) Enteric neurons show a primary cilium. J Cell Mol Med $17: 147-153$

Luo J, Chen X, Pan YW, Lu S, Xia Z, Storm DR (2015) The type 3 adenylyl cyclase is required for the survival and maturation of newly generated granule cells in the olfactory bulb. PLoS One 10:e0122057.

Luskin MB (1993) Restricted proliferation and migration of postnatally generated neurons derived from the forebrain subventricular zone. Neuron 11:173-189.

Madisen L, Zwingman TA, Sunkin SM, Oh SW, Zariwala HA, Gu H, Ng LL, Palmiter RD, Hawrylycz MJ, Jones AR, Lein ES, Zeng H (2010) A robust and high-throughput cre reporting and characterization system for the whole mouse brain. Nat Neurosci 13:133-140.

Malicki JJ, Johnson CA (2017) The cilium: cellular antenna and central processing unit. Trends Cell Biol 27:126-140.

Mazo G, Soplop N, Wang WJ, Uryu K, Tsou MF (2016) Spatial control of primary ciliogenesis by subdistal appendages alters sensation-associated properties of cilia. Dev Cell 39:424-437.

Menn B, Garcia-Verdugo JM, Yaschine C, Gonzalez-Perez O, Rowitch D, Alvarez-Buylla A (2006) Origin of oligodendrocytes in the subventricular zone of the adult brain. J Neurosci 26:7907-7918.

Monsma FJ Jr, Shen Y, Ward RP, Hamblin MW, Sibley DR (1993) Cloning and expression of a novel serotonin receptor with high affinity for tricyclic psychotropic drugs. Mol Pharmacol 43:320-327.

Nakamuta S, Yang YT, Wang CL, Gallo NB, Yu JR, Tai Y, Van Aelst L (2017) Dual role for DOCK7 in tangential migration of interneuron precursors in the postnatal forebrain. J Cell Biol 216:4313-4330.

Nguyen HB, Thai TQ, Saitoh S, Wu B, Saitoh Y, Shimo S, Fujitani H, Otobe H, Ohno N (2016) Conductive resins improve charging and resolution of acquired images in electron microscopic volume imaging. Sci Rep 6:23721.

Obernier K, Alvarez-Buylla A (2019) Neural stem cells: origin, heterogeneity and regulation in the adult mammalian brain. Development 146 : dev156059.

Ogino T, Sawada M, Takase H, Nakai C, Herranz-Perez V, Cebrian-Silla A, Kaneko N, Manuel Garcia-Verdugo J, Sawamoto K (2016) Characterization of multiciliated ependymal cells that emerge in the neurogenic niche of the aged zebrafish brain. J Comp Neurol 524:2982-2992.

Ohno N, Katoh M, Saitoh Y, Saitoh S, Ohno S (2015) Three-dimensional volume imaging with electron microscopy toward connectome. Microscopy (Oxf) 64:17-26.

Ota H, Hikita T, Sawada M, Nishioka T, Matsumoto M, Komura M, Ohno A, Kamiya Y, Miyamoto T, Asai N, Enomoto A, Takahashi M, Kaibuchi K, Sobue K, Sawamoto K (2014) Speed control for neuronal migration in the postnatal brain by Gmip-mediated local inactivation of RhoA. Nat Commun 5:4532.
Parent JM, Vexler ZS, Gong C, Derugin N, Ferriero DM (2002) Rat forebrain neurogenesis and striatal neuron replacement after focal stroke. Ann Neurol 52:802-813.

Paridaen JT, Wilsch-Bräuninger M, Huttner WB (2013) Asymmetric inheritance of centrosome-associated primary cilium membrane directs ciliogenesis after cell division. Cell 155:333-344.

Pazour GJ, Dickert BL, Vucica Y, Seeley ES, Rosenbaum JL, Witman GB, Cole DG (2000) Chlamydomonas IFT88 and its mouse homologue, polycystic kidney disease gene tg737, are required for assembly of cilia and flagella. J Cell Biol 151:709-718.

Petreanu L, Alvarez-Buylla A (2002) Maturation and death of adult-born olfactory bulb granule neurons: role of olfaction. J Neurosci 22:61066113.

Ponti G, Obernier K, Guinto C, Jose L, Bonfanti L, Alvarez-Buylla A (2013) Cell cycle and lineage progression of neural progenitors in the ventricular-subventricular zones of adult mice. Proc Natl Acad Sci U S A 110:E1045-E1054.

Riccio O, Potter G, Walzer C, Vallet P, Szabó G, Vutskits L, Kiss JZ, Dayer AG (2009) Excess of serotonin affects embryonic interneuron migration through activation of the serotonin receptor 6. Mol Psychiatry 14:280-290.

Robert A, Margall-Ducos G, Guidotti JE, Brégerie O, Celati C, Bréchot C, Desdouets C (2007) The intraflagellar transport component IFT88/polaris is a centrosomal protein regulating $\mathrm{G}_{1}-\mathrm{S}$ transition in non-ciliated cells. J Cell Sci 120:628-637.

Ruat M, Traiffort E, Arrang JM, Tardivel-Lacombe J, Diaz J, Leurs R, Schwartz JC (1993) A novel rat serotonin (5-HT6) receptor: molecular cloning, localization and stimulation of cAMP accumulation. Biochem Biophys Res Commun 193:268-276.

Saghatelyan A, de Chevigny A, Schachner M, Lledo PM (2004) Tenascin-R mediates activity-dependent recruitment of neuroblasts in the adult mouse forebrain. Nat Neurosci 7:347-356.

Sawada M, Ohno N, Kawaguchi M, Huang SH, Hikita T, Sakurai Y, Bang Nguyen H, Quynh Thai T, Ishido Y, Yoshida Y, Nakagawa H, Uemura A, Sawamoto K (2018) PlexinD1 signaling controls morphological changes and migration termination in newborn neurons. EMBO J 37:e97404.

Sawamoto K, Wichterle H, Gonzalez-Perez O, Cholfin JA, Yamada M, Spassky N, Murcia NS, Garcia-Verdugo JM, Marin O, Rubenstein JL, Tessier-Lavigne M, Okano H, Alvarez-Buylla A (2006) New neurons follow the flow of cerebrospinal fluid in the adult brain. Science 311:629632.

Schaar BT, McConnell SK (2005) Cytoskeletal coordination during neuronal migration. Proc Natl Acad Sci U S A 102:13652-13657.

Schrøder JM, Larsen J, Komarova Y, Akhmanova A, Thorsteinsson RI, Grigoriev I, Manguso R, Christensen ST, Pedersen SF, Geimer S, Pedersen LB (2011) EB1 and EB3 promote cilia biogenesis by several centrosomerelated mechanisms. J Cell Sci 124:2539-2551.

Seki T, Arai Y (1991) The persistent expression of a highly polysialylated NCAM in the dentate gyrus of the adult rat. Neurosci Res 12:503-513.

Seligman AM, Wasserkrug HL, Hanker JS (1966) A new staining method (OTO) for enhancing contrast of lipid-containing membranes and droplets in osmium tetroxide-fixed tissue with osmiophilic thiocarbohydrazide (TCH) . J Cell Biol 30:424-432.

Shinohara R, Thumkeo D, Kamijo H, Kaneko N, Sawamoto K, Watanabe K, Takebayashi H, Kiyonari H, Ishizaki T, Furuyashiki T, Narumiya S (2012) A role for mDia, a rho-regulated actin nucleator, in tangential migration of interneuron precursors. Nat Neurosci 15:373-380, S1-2.

Smith JW, Christie KN, Frame J (1969) Desmosomes, cilia and acanthosomes associated with keratocytes. J Anat 105:383-392.

Sorokin S (1962) Centrioles and the formation of rudimentary cilia by fibroblasts and smooth muscle cells. J Cell Biol 15:363-377.

Teng J, Rai T, Tanaka Y, Takei Y, Nakata T, Hirasawa M, Kulkarni AB, Hirokawa N (2005) The KIF3 motor transports N-cadherin and organizes the developing neuroepithelium. Nat Cell Biol 7:474-482.

Thai TQ, Nguyen HB, Saitoh S, Wu B, Saitoh Y, Shimo S, Elewa YH, Ichii O, Kon Y, Takaki T, Joh K, Ohno N (2016) Rapid specimen preparation to improve the throughput of electron microscopic volume imaging for three-dimensional analyses of subcellular ultrastructures with serial block-face scanning electron microscopy. Med Mol Morphol 49: $154-162$. 
Tong CK, Han YG, Shah JK, Obernier K, Guinto CD, Alvarez-Buylla A (2014) Primary cilia are required in a unique subpopulation of neural progenitors. Proc Natl Acad Sci U S A 111:12438-12443.

Trivedi N, Ramahi JS, Karakaya M, Howell D, Kerekes RA, Solecki DJ (2014) Leading-process actomyosin coordinates organelle positioning and adhesion receptor dynamics in radially migrating cerebellar granule neurons. Neural Dev 9:26.

Tsai JW, Bremner KH, Vallee RB (2007) Dual subcellular roles for LIS1 and dynein in radial neuronal migration in live brain tissue. Nat Neurosci 10:970-979.

Watanabe T, Kakeno M, Matsui T, Sugiyama I, Arimura N, Matsuzawa K, Shirahige A, Ishidate F, Nishioka T, Taya S, Hoshino M, Kaibuchi K (2015) TTBK2 with EB1/3 regulates microtubule dynamics in migrating cells through KIF2A phosphorylation. J Cell Biol 210:737-751.
Wheway G, Nazlamova L, Hancock JT (2018) Signaling through the primary cilium. Front Cell Dev Biol 6:8.

Willingham MC, Rutherford AV (1984) The use of osmium-thiocarbohydrazide-osmium (OTO) and ferrocyanide-reduced osmium methods to enhance membrane contrast and preservation in cultured cells. J Histochem Cytochem 32:455-460.

Yamaguchi M, Mori K (2005) Critical period for sensory experiencedependent survival of newly generated granule cells in the adult mouse olfactory bulb. Proc Natl Acad Sci U S A 102:9697-9702.

Yamashita T, Ninomiya M, Hernández Acosta P, García-Verdugo JM, Sunabori T, Sakaguchi M, Adachi K, Kojima T, Hirota Y, Kawase T, Araki N, Abe K, Okano H, Sawamoto K (2006) Subventricular zone-derived neuroblasts migrate and differentiate into mature neurons in the post-stroke adult striatum. J Neurosci 26:6627-6636. 